\title{
Polycomb repressive complex 2 is a critical mediator of allergic inflammation
}

\author{
Christine R. Keenan, ${ }^{1,2}$ Nadia lannarella, ${ }^{1}$ Alexandra L. Garnham, ${ }^{1,2}$ Alexandra C. Brown, ${ }^{3}$ \\ Richard Y. Kim, ${ }^{3}$ Jay C. Horvat, ${ }^{3}$ Philip M. Hansbro, ${ }^{3,4}$ Stephen L. Nutt, ${ }^{1,2}$ and Rhys S. Allan ${ }^{1,2}$ \\ 'The Walter and Eliza Hall Institute of Medical Research, Parkville, Victoria, Australia. ${ }^{2}$ Department of Medical Biology, \\ The University of Melbourne, Parkville, Victoria, Australia. ${ }^{3}$ Priority Research Centre for Healthy Lungs, Hunter Medical \\ Research Institute and the University of Newcastle, Newcastle, Australia. ${ }^{4}$ Centre for Inflammation, Centenary Institute \\ and University of Technology Sydney, Sydney, Australia.
}

\begin{abstract}
Strategies that intervene with the development of immune-mediated diseases are urgently needed, as current treatments mostly focus on alleviating symptoms rather than reversing the disease. Targeting enzymes involved in epigenetic modifications to chromatin represents an alternative strategy that has the potential to perturb the function of the lymphocytes that drive the immune response. Here, we report that 2 major epigenetic silencing pathways are increased after $T$ cell activation. By specific inactivation of these molecules in the T cell compartment in vivo, we demonstrate that the polycomb repressive complex 2 (PRC2) is essential for the generation of allergic responses. Furthermore, we show that small-molecule inhibition of the PRC2 methyltransferase, enhancer of zeste homolog 2 (Ezh2), reduces allergic inflammation in mice. Therefore, by systematically surveying the pathways involved in epigenetic gene silencing we have identified Ezh2 as a target for the suppression of allergic disease.
\end{abstract}

Authorship note: SLN and RSA are co-senior authors.

Conflict of interest: CRK, SLN, and RSA are inventors on a patent application (Australian provisional patent application no. 2018904884) about inhibiting PRC2 to treat inflammation.

Copyright: (c) 2019 American Society for Clinical Investigation

Submitted: February 2, 2019

Accepted: April 17, 2019

Published: May 16, 2019

Reference information: /CI Insight. 2019;4(10):e127745. https://doi. org/10.1172/ji.insight.127745.

\section{Introduction}

The immune system functions to maintain homeostasis and protect against pathogen challenge. However, sometimes hypersensitivity to usually innocuous antigens results in allergic conditions such as asthma and allergic rhinitis or autoinflammatory diseases such as lupus and type 1 diabetes. Unfortunately, these disorders are increasing in prevalence and current treatments are mostly aimed at ameliorating the symptoms but not switching off the lymphocytes that cause the disease pathology. Therefore, novel strategies are desperately needed to rewire the immune response in such situations with the aim to perturb the inflammatory cascade and potentially cure disease.

The major orchestrators of immune cell function in the development of allergic responses are the $\mathrm{CD}^{+} \mathrm{T}$ helper (Th) cells. In animal models of allergic asthma, the presence of $\mathrm{CD}^{+} \mathrm{T}$ cells is absolutely required for the induction of airway inflammation and recruitment of eosinophils (1). Allergen-specific Th cells are activated by dendritic cells in the draining lymph nodes and infiltrate into the lung tissue and secrete cytokines to orchestrate and exacerbate the inflammatory response (2). Although a number of Th subsets are known, the main players in promoting this allergic response appear to be the Th2 subset, which produces important cytokines such as IL- $4,-5$, and -13 and chemokine signals that result in the recruitment and activation of the innate immune cells.

The factors that control the relative stability and plasticity of Th phenotypes are critical to immune responses (3-5), and although the signals and transcription factors guiding Th differentiation are well defined, it appears that the heritability through cell division is controlled predominantly by epigenetic mechanisms. Correlations have been observed between levels of several histone $\mathrm{H} 3$ and $\mathrm{H} 4$ modifications and activation or silencing of cytokine genes in committed Th1 and Th2 cells (6). For example, differentiation to a Th1 phenotype requires epigenetic silencing of genes encoding Th2 cytokines such as IL- 4 and IL-13 (7-9). Conversely, differentiation to a Th2 phenotype requires epigenetic silencing of Th1 cytokine genes such as the gene encoding IFN- $\gamma(9,10)$. Importantly, we have found that stable Th1 gene silencing in Th2 cells required the expression of the Suv39h1 enzyme to maintain the balance of methylation and acetylation at lysine 9 of histone H3 (H3K9) in Th1 genes (10). Furthermore, in a murine model of Th2 allergic asthma, the loss of Suv39h1 resulted in skewing towards a Th1 response and decreased lung pathology (10). 
Targeting enzymes involved in epigenetic modifications of chromatin therefore has the potential to perturb the function of the lymphocytes that drive the allergic response. However, it is unknown whether other epigenetic modifiers or pathways are also involved in the allergic response.

In this study, we searched for epigenetic pathways that are critical for T cells to orchestrate the development of allergic inflammation. We identified components involved in epigenetic gene silencing that were upregulated after $\mathrm{T}$ cell activation and performed in vivo inactivation of these molecules specifically in the $\mathrm{T}$ cell lineage. These experiments revealed that the polycomb repressive complex 2 (PRC2) pathway was essential for the development of allergic inflammation. We then build on these discoveries to show that small-molecule inhibition of Ezh2 function represents a promising strategy for suppressing the development of allergic disease.

\section{Results}

Identification of heterochromatin components upregulated during $C D 4^{+} T$ cell activation. We hypothesized that those chromatin-associated proteins which play important regulatory roles in the allergic immune response and as such represent potential therapeutic targets would be upregulated after T cell activation. To identify such molecules, we examined the expression of genes encoding 34 different repressive chromatin components associated with histone modification in publicly available microarray data comparing nonstimulated human $\mathrm{CD}^{+} \mathrm{T}$ cells to those stimulated for 24 hours (11). We observed that molecules associated with the PRC2/H3K27me3 (EZH2, EED, and RBBP7) and Suv39h/HP1/H3K9me3 (SUV39H1, SUV39H2, CBX1, $C B X 3, C B X 5$, and TRIM28) pathways were significantly upregulated (adjusted $P<0.05$ ) in response to T cell activation, whereas other components such as PRC1 members (BMI1, RING1, CBX2, -4, -7, and -8), most HDACs (excluding HDAC2), and other lysine methyltransferases such as DOT1L, EZH1, EHMT1 and -2, and SETDB1 were not significantly altered or even downregulated (Figure 1A). Thus, components of 2 major epigenetic silencing pathways shown in Figure 1D are upregulated after T cell activation.

We then examined the activation-induced regulation of these chromatin-modifying genes at the protein level. We activated wild-type (WT) mouse $\mathrm{CD}^{+} \mathrm{T}$ cells and then performed immunoblotting to assess the levels of each protein for which reliable antibodies were available. In line with the microarray analysis we observed substantially increased protein levels of the PRC2 components Ezh2 and Suz12 in cells activated for 24 hours (Figure 1, B and C). We also observed increased levels of HP1 $\alpha(C b \times 5)$ and HP1 $\beta(C b \times 1)$ protein levels, whereas the expression of the HP1 binding factor, transcription intermediary factor 1- $\beta$ (TIF1 $\beta$; encoded by Trim28) was unaltered at this time point (Figure 1, B and C). Levels of the repressive histone marks $\mathrm{H} 3 \mathrm{~K} 9 \mathrm{me} 3$ and $\mathrm{H} 3 \mathrm{~K} 27 \mathrm{me} 3$ mirror the results for the corresponding enzymatic complexes where activated cells showed significantly higher global levels than naive cells (Figure 1, B and C). LaminB1 was used as a nuclear loading control and was found to be lower in activated samples (Figure 1B), suggesting we may be underestimating the nuclear upregulation of the protein components of these epigenetic silencing pathways. Based on these data, we next chose to explore the importance of the PRC2 pathway and corepressors of the Suv39h/H3K9me3 pathway in allergic asthma by specifically inactivating them in $\mathrm{T}$ cells.

Auxiliary components of the Suv39h/H3K9me3/HP1 pathway are not required for the development of allergic inflammation. Given that a number of molecules in the Suv39h1/H3K9me3/HP1 pathway were upregulated after T cell activation (Figure 1), and our previous work implicating Suv39h1 in the development of allergic asthma (10), we examined the role of $\mathrm{HP} 1 \alpha, \mathrm{HP} 1 \beta$, and TIF1 $\beta$ in the allergic response. Previously, we had implicated HP1 $\alpha(C b \times 5)$ but not HP1 $\gamma(C b \times 3)$ in Th2 cell stability (10), making HP1 $\gamma$ an unlikely candidate. Therefore, mice that had exons of $C b x 5$ (HP1 $\alpha), C b x 1$ (HP1 $\beta)$, or Trim 28 (TIF1 $\beta$ ) flanked by loxP sequences were crossed with transgenic mice expressing Cre recombinase under the control of the $C d 4$ promoter. In order to confirm the loss of the gene product specifically in $\mathrm{T}$ cells in each of these strains, we sorted splenic $\mathrm{B}$ cells $\left(\mathrm{B} 220^{+}\right)$and $\mathrm{CD} 4^{+} \mathrm{T}$ cells $\left(\mathrm{TCR} \beta^{+}, \mathrm{CD} 4^{+}\right)$(Supplemental Figure 1A; supplemental material available online with this article; https://doi.org/10.1172/jci.insight.127745DS1), and examined HP1 $\alpha, \mathrm{HP} 1 \beta$, and TIF1 $\beta$ protein expression by Western blotting (Supplemental Figure 1B). Further analysis revealed that all these mice displayed normal $\mathrm{T}$ cell development (data not shown). To test the ability of these mice to mount an allergic response we subjected them to the classical ovalbumin (OVA) challenge model prior to comprehensive analysis of the cellular and cytokine composition of the lung environment. In this model, mice are first sensitized to OVA in the presence of the adjuvant aluminum hydroxide (alum) prior to challenge with aerosolized OVA. Despite lacking these molecules, surprisingly, these mice had normal cellular infiltrate and developed allergic pathology equivalent to their littermate counterparts (Supplemental Figure 2, A-D). 

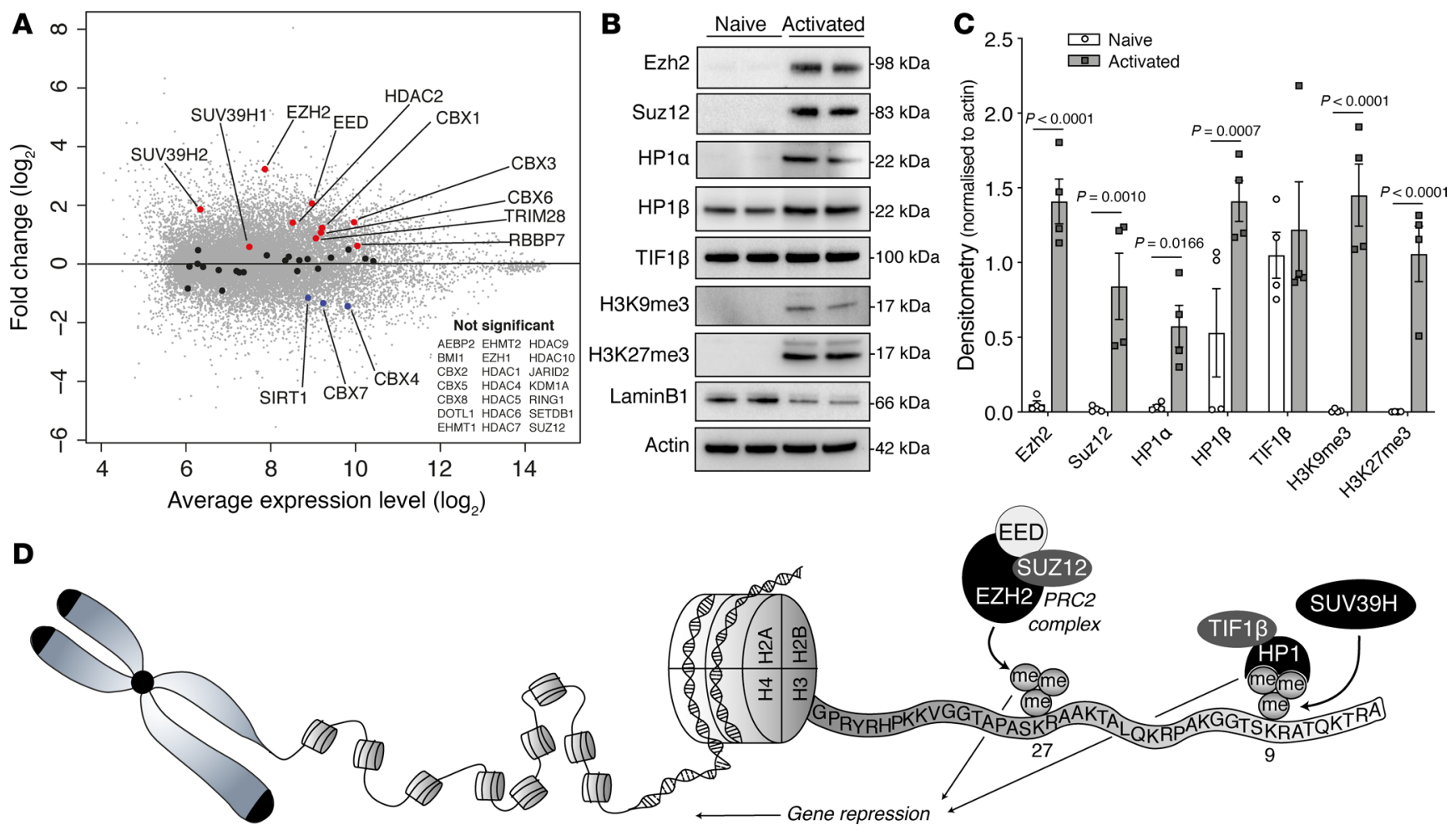

Figure 1. Components of the PRC2 and Suv39h/HP1 pathways are specifically upregulated after T cell activation. (A) Quantitation of the expression changes of the genes encoding 34 repressive chromatin components associated with histone modification from publicly available human CD4 ${ }^{+} \mathrm{T}$ cell microarray data (naive vs. 24-hour activation with anti-CD3 and anti-CD28) (11). Genes significantly upregulated are denoted in red, genes significantly downregulated are denoted in blue, and genes not significantly altered are denoted in black. Other genes are denoted in gray. (B) Representative Western blots of naive versus 24-hour-activated (anti-CD3 and anti-CD28) C57BL/6 mouse CD4 ${ }^{+} T$ cells (biological replicates shown) with quantification (mean \pm SEM with individual data points from 4 samples) shown (C). Statistical significance was determined by 2-way ANOVA with Holm-Sidak post hoc test. (D) Schematic displaying the molecules involved in the H3K27me3-associated PRC2 and H3K9me3-associated Suv39h/HP1 gene silencing.

In order to exclude confounding influences from background effects we performed bronchoalveolar lavage (BAL) on naive $C b x 5^{\mathrm{f} / \mathrm{fl}} C d 4^{\mathrm{Cre}}, C b x 1^{\mathrm{fl} / \mathrm{fl}} C d 4^{\mathrm{Cre}}$, and Trim $28^{\mathrm{H} / \mathrm{fl}} C d 4^{\mathrm{Cre}}$ mice and their floxed counterparts and found no difference in individual leukocyte populations within the lung of deleted or floxed mice, with alveolar macrophages forming the largest population of cells, as expected (Supplemental Figure 3). Bio-Plex cytokine analysis of the acellular lavage fluid from OVA-exposed mice showed some alterations in cytokine levels (Supplemental Figure 2E), particularly IL-4 and IL-5 levels in $C b x 5^{\mathrm{n} / \mathrm{fl}} C d 4^{\mathrm{Cre}}$ mice; however, this did not result in decreased inflammatory infiltration or decreased lung pathology (Supplemental Figure 2, A-D). Thus, HP1 $\alpha$, HP1 $\beta$, and TIF1 $\beta$ molecules are not critical for T cells to drive allergic inflammation.

$T$ cells rely on PRC2 components to drive allergic inflammation. We then turned our focus to the role of Ezh2 in the allergic response, as it was the most upregulated gene and protein following $\mathrm{T}$ cell activation. We bred mice in which the exons of Ezh2 (12) are flanked by loxP sequences with transgenic mice expressing Cre recombinase under the control of the $C d 4$ promoter (13). We have previously published that this results in efficient deletion of Ezh2 in the T cell lineage and that these mice display normal $\mathrm{T}$ cell development, but have alterations in $\mathrm{CD}^{+}$memory phenotypes and NKT cell expansion (14). Therefore, as a control we exposed some mice to alum alone prior to OVA challenge to test for any preexisting or spontaneous immune reaction driven by the gene deficiency (Figure 2A). We first examined the BAL infiltrate by flow cytometry. $E z h 2^{\mathrm{f} / \mathrm{fl}} C d 4^{\mathrm{Cre}}$ mice were completely protected from eosinophil, neutrophil, and T cell infiltration into the airways following OVA challenge (Figure 2, B-D). The forward scatter (FSC) versus side scatter (SSC) profiles of the neutrophil, eosinophil, alveolar macrophage, and $\mathrm{T}$ cell populations gated in Figure 2C can be seen in Supplemental Figure 4. Each of these populations correspond to the major cellular populations visible on FSC versus SSC plots shown in Figure 2B. Cells recovered from these Ezh2t/fl $C d 4^{\text {re }}$ mice were predominantly alveolar macrophages (FSC ${ }^{\text {hi }}, \mathrm{SSC}^{\text {hi }}, \mathrm{CD}_{11} \mathrm{~b}^{+}, \mathrm{CD}_{11 \mathrm{c}^{+}}, \mathrm{SiglecF}^{+}$), a resident, noninflammatory cell population whose numbers were consistent across groups. 
A

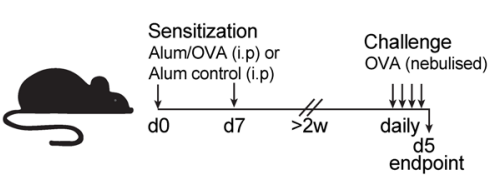

C

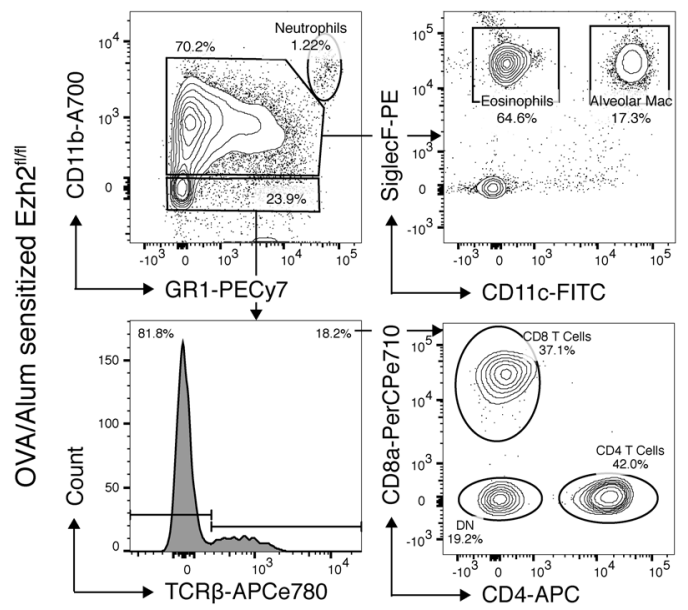

D

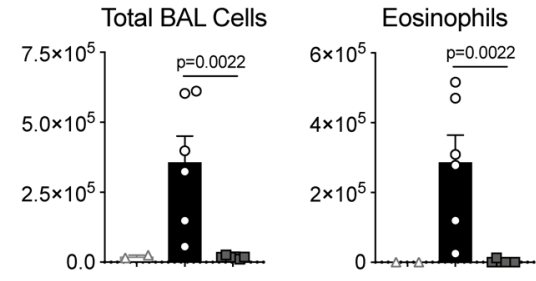

E

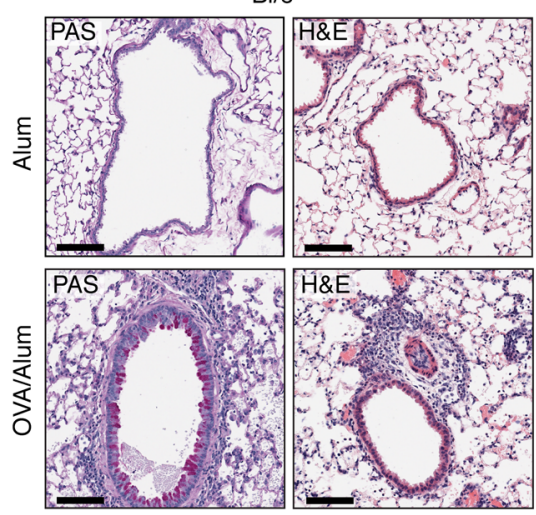

G

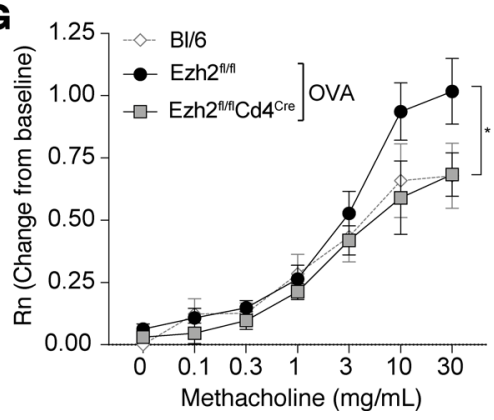

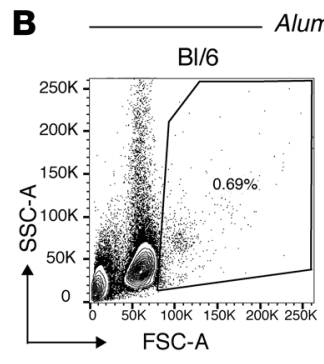
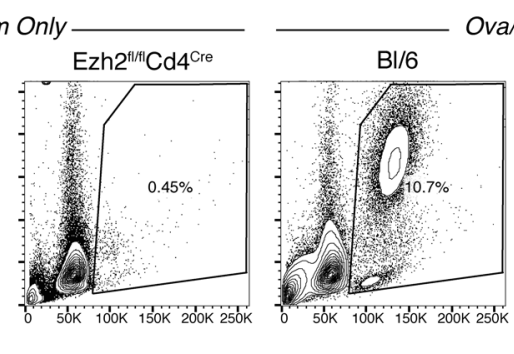

Ova/Alum
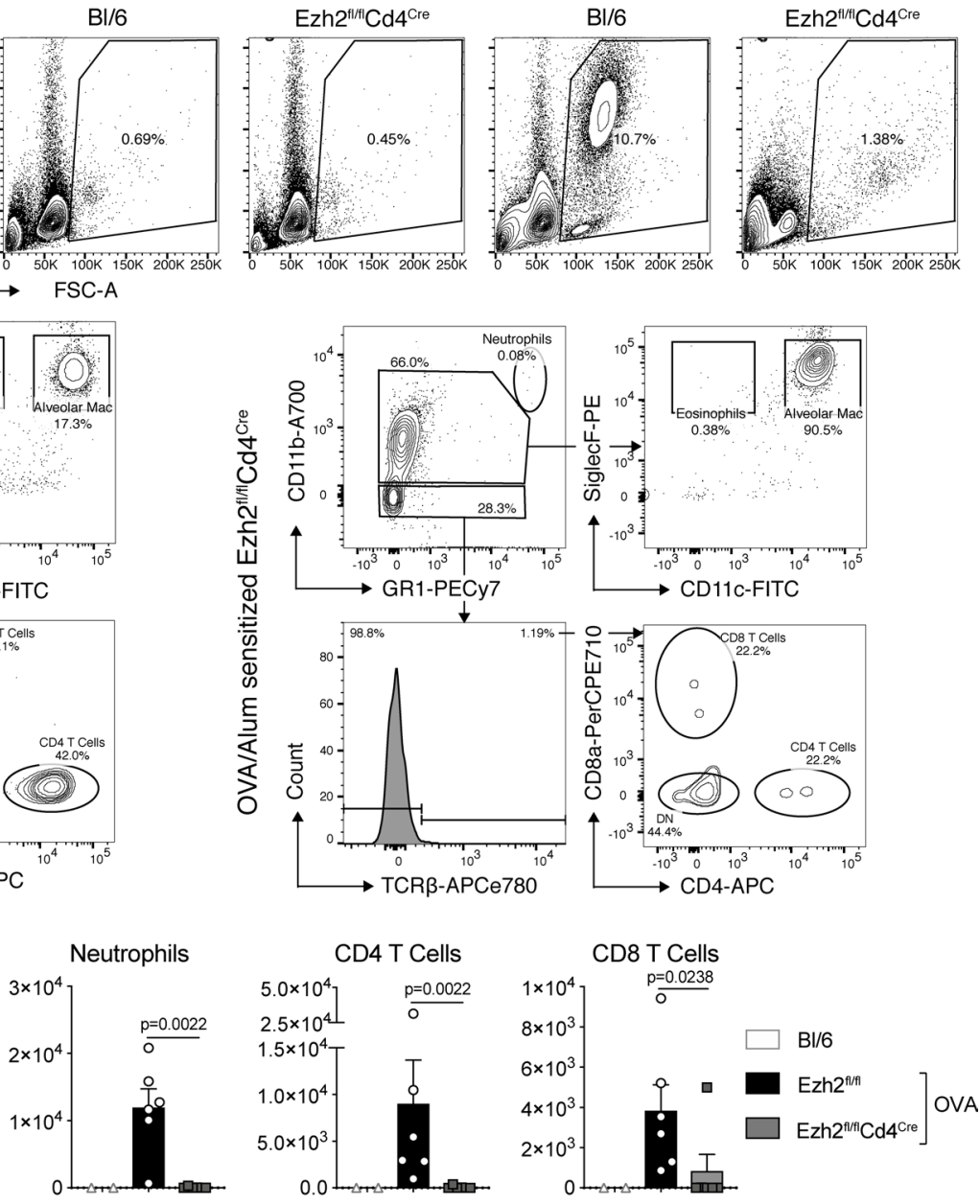

Ezh2 $2^{\text {flltit }} \mathrm{Cd} 4^{\mathrm{Cre}}$
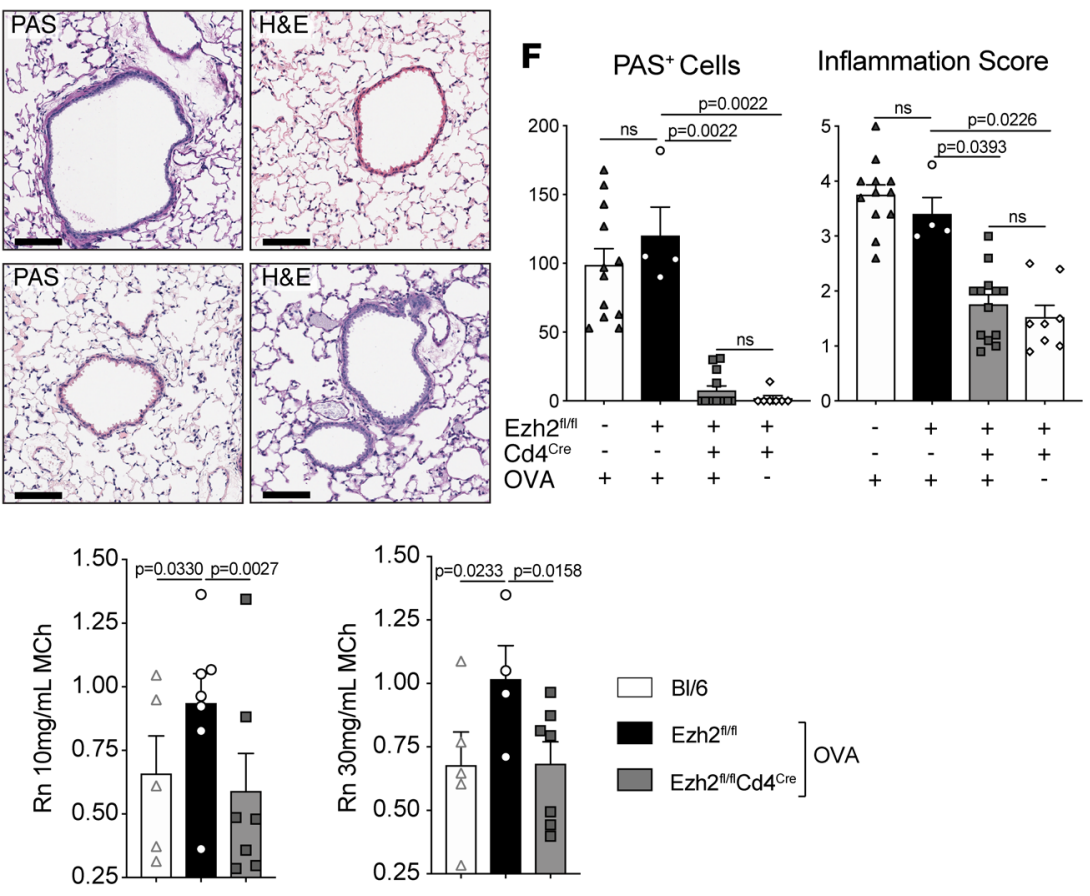
Figure 2. Ezh2 is essential for T cells to drive allergic inflammation. (A) Experimental protocol of OVA-induced allergic inflammation. (B) Representative forward-scatter (FSC) versus side-scatter (SSC) flow cytometric plots of bronchoalveolar lavage (BAL) samples from Ezh2 $2^{\mathrm{fl} / \mathrm{fl}} \mathrm{Cd} 4^{\mathrm{Cre}}$ and WT C57BL/6 (BI/6) mice following OVA-induced allergic inflammation. Data shown are representative of $n=2$ (WT/alum), $n=8$ (Ezh2 $2^{\text {fl/fl }}$ Cd4 $4^{\text {Cre }} /$ alum), $n=12$ (WT/OVA), $n=11$

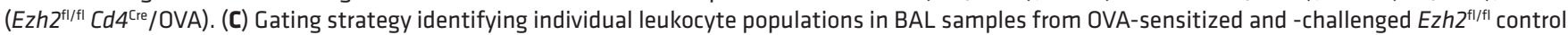

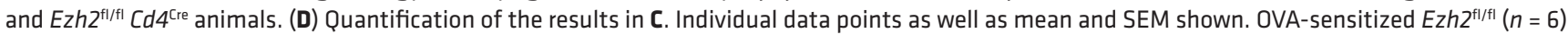
and $E z h 2^{\mathrm{fl} / \mathrm{fl}} C d 4^{\mathrm{Cre}}(n=6)$ groups were compared by Mann-Whitney $U$ test. Unchallenged BI/6 $(n=2)$ shown for comparative purposes. (E) Representative histological analysis of the airways of $E z h 2^{\text {fl/fl }} C D 4^{\text {Cre }}$ and BI/6 mice following OVA-induced allergic inflammation. Scale bars: $100 \mu \mathrm{m}$. (F) Quantification of $\mathrm{PAS}^{+}$cells and inflammation score. OVA refers to initial sensitization with all mice exposed to nebulized OVA challenge. Mean and SEM as well as individ-

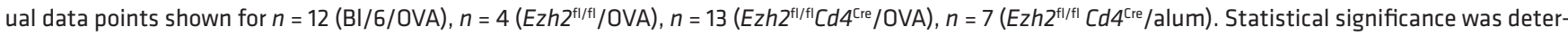
mined by Kruskal-Wallis $H$ test with Dunn's post hoc test. (G) Airway resistance (Rn) following OVA-induced allergic inflammation in Ezh2 ${ }^{\mathrm{fl} / \mathrm{fl}}(\mathrm{n}=7 \mathrm{for} 0-10$ $\mathrm{mg} / \mathrm{ml}$ methacholine [MCh], $n=6$ for $30 \mathrm{mg} / \mathrm{ml} \mathrm{MCh})$ and Ezh2 ${ }^{\mathrm{fl} / \mathrm{fl}} C d 4^{\text {Cre }}$ mice $(n=7)$. Unchallenged Bl $/ 6$ mice $(n=6$ for $0-3 \mathrm{mg} / \mathrm{ml} \mathrm{MCh,} n=5$ for $10-30$ $\mathrm{mg} / \mathrm{ml} \mathrm{MCh}$ ) were included to indicate baseline bronchoconstriction in response to MCh. Mean and SEM together with individual data points are shown. Data were analyzed by 2-way ANOVA with Bonferroni's post hoc test. ${ }^{*} P<0.05$ with specific comparisons denoted in central and right-hand panels.

We next examined the lung histopathology following OVA challenge in $E z h 2^{\text {2//1 }} C d 4^{\text {Cre }}$ and control mice. OVA-sensitized $E z h 2^{\text {2//1/ }} C d 4^{\text {cre }}$ mice were completely protected from OVA-induced increases in PAS ${ }^{+}$ mucous-producing cells and lung tissue inflammation levels (Figure 2, E and F), statistically indistinguishable from alum-only sensitized control mice (Figure 2, E and F), demonstrating that Ezh2 deletion in T cells can completely prevent lung inflammation and mucous hypersecretion, characteristic of pulmonary allergic inflammation. We next sought to examine whether this protection from pulmonary inflammation translated to improvements in lung function. Using the forced-oscillation technique coupled with inhaled bronchoconstrictor agent (methacholine, $0.1-30 \mathrm{mg} / \mathrm{ml}$ ), we found that OVA-induced hyperresponsiveness seen in $E z h 2^{\mathrm{n} / \mathrm{fl}}$ control mice was absent in $E z h 2^{\mathrm{n} / \mathrm{ll}} C d 4^{\mathrm{Cre}}$ mice whose airway resistance (Rn) was indistinguishable from unchallenged C57BL/6 WT mice (Figure 2G).

We also used an alternative model of allergic inflammation representing a more physiologically relevant allergen, house dust mite (HDM) extract (Supplemental Figure 5A). In line with the previous findings using the OVA model, we found $E z h 2^{\mathrm{a} / \mathrm{ll}} C d 4^{\mathrm{Cre}}$ mice to be completely protected from the development of HDM-induced allergic inflammation (Supplemental Figure 5, B-E). HDM-induced increases in BAL leukocytes in $E z h 2^{\mathrm{A} / \mathrm{ll}}$ control mice were significantly reduced in $E z h 2^{\mathrm{A} / \mathrm{ll}} C d 4^{\mathrm{Cre}}$ mice, with the cell numbers recovered from $E z h 2^{1 / 1 / l} C d 4^{\text {Cre }}$ BAL indistinguishable from PBS-exposed controls (Supplemental Figure 5C). As expected, the cellular infiltrate was predominantly eosinophils and $\mathrm{T}$ cells, with a lack of robust neutrophil accumulation in this model (Supplemental Figure 5C). Lung histopathology showed reduced mucous-producing cells and decreased lung inflammation levels in HDM-challenged $E z h 2^{\mathrm{A} / \mathrm{l}} \mathrm{Cd} 4^{\mathrm{Cre}}$ mice compared with $E z h 2^{\mathrm{n} / \mathrm{ll}}$ controls (Supplemental Figure 5, D and E), again consistent with previous results using the OVA model. Overall, these results suggest that the presence of Ezh2 in T cells is absolutely required for allergic responses in the airways.

In order to confirm that Ezh2 is mediating allergic inflammation through its canonical role as the enzymatic component of the PRC2 complex, we also separately inactivated the nonredundant core components of PRC2, Suz12 and Eed, specifically in T cells. Importantly, EED was the second most highly upregulated repressive component after EZH2 in the T cell activation human microarray data set (Figure 1A), and Suz12 was highly upregulated in mouse T cells at the protein level (Figure 1C). Using mice deficient in either

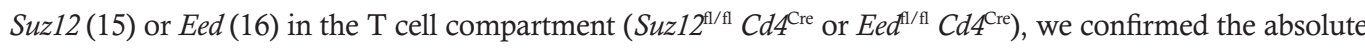
requirement for these molecules in the generation of allergic histopathology (Supplemental Figure 6A), eosinophilia (Supplemental Figure 6, B and C), and cytokine production (Supplemental Figure D) in the lungs of OVA-challenged mice. Thus, we confirm that the PRC2 pathway is critical to development of T cell allergy.

Ezh2 is required to generate antigen-specific memory. We next wanted to investigate the mechanism by which deletion of PRC2 components in T cells prevents allergic inflammation. We first examined cytokine levels in the acellular BAL fluid following OVA challenge and found almost all OVA-induced cytokines including the Th2 cytokines IL- 4 and IL- 5 and the proinflammatory cytokines IL- 6 and KC (the murine CXCL8 homolog) to be reduced to baseline levels in the BAL fluid from $E z h 2^{\mathrm{A} / \mathrm{ll}} \mathrm{Cd} 4^{\text {Cre }}$ mice (Figure $3 \mathrm{~A}$ ). Importantly, loss of Ezh2 did not result in a skewing towards Th1 cytokines in the BAL fluid, with IFN- $\gamma$ levels consistent across all groups (Figure 3B).

We next quantified the titers of OVA-specific and total IgE in serum from these mice following OVA challenge. We found that $E z h 2^{\text {I/f }} C d 4^{\text {re }}$ mice lacked any detectable OVA-specific IgE above the background of the assay (Figure 3C), despite normal levels of serum total IgE (Figure 3C). Detectable levels of OVA-specific IgE were found in the serum from C57BL/6 control mice following OVA challenge, as expected (Figure 3C). 

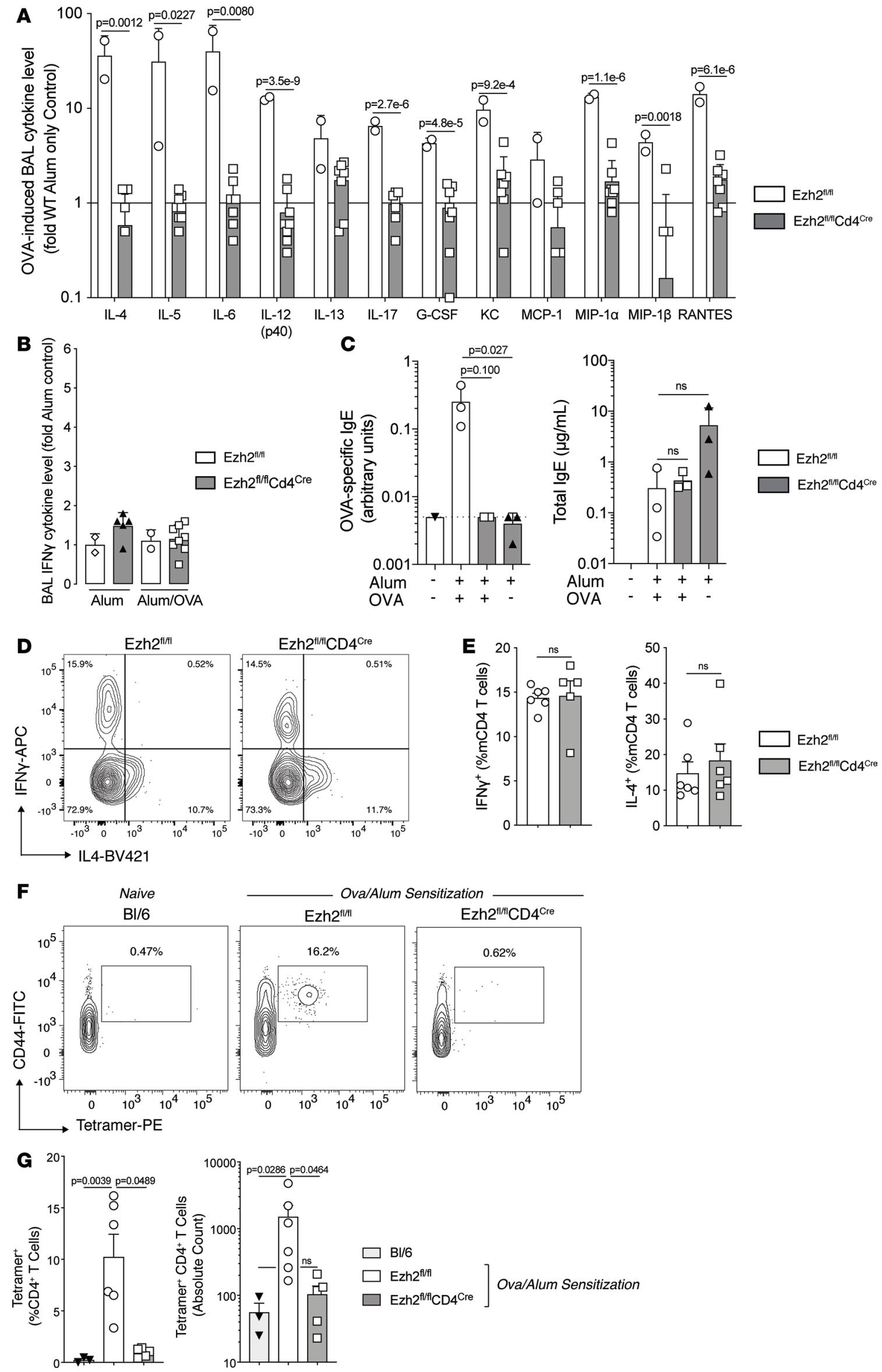

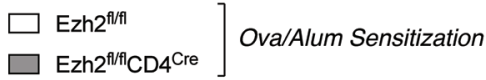


Figure 3. Ezh2 is required to generate antigen-specific memory. (A) Bio-Plex analysis of cytokine levels in bronchoalveolar lavage (BAL) fluid following

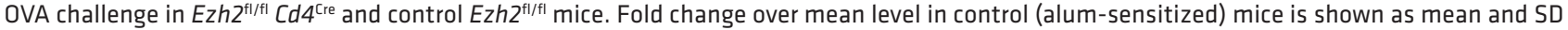
together with individual data points. Statistical analysis by multiple unpaired $t$ tests corrected for multiple comparisons using the Holm-Sidak method;

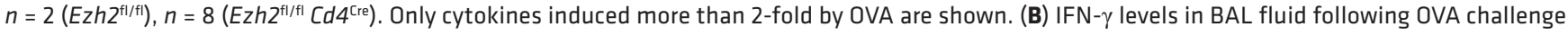
The $x$-axis labels indicate initial sensitization. Fold change over mean of alum-sensitized control mice shown as individual data points together with mean and SD. Statistical analysis by 2-way ANOVA. Group sizes are as in A. (C) OVA-specific IgE and total IgE detected in serum of mice following OVA challenge. The $x$-axis labels indicate initial sensitization. Individual data points as well as mean and SD are shown; $n=3$ per group except for nonimmunized C57BL/6 (BI/6), where $n=1$ is shown for reference. Statistical analysis by Kruskal-Wallis $H$ test with Dunn's post hoc test. (D) Intracellular cytokine staining of splenic antigen-experienced (CD44 $\left.{ }^{+}\right) C D^{+} T$ cells 3 days after OVA sensitization protocol (10 days after initial sensitization). Cells were stimulated with PMA/ionomycin for 5 hours in the presence of GolgiStop protein transport inhibitor for the final 2 hours. (E) Quantification of the results in $\mathbf{D}$. Individual data points together with mean and SEM are shown. Statistical analysis by Mann-Whitney $U$ test ( $n=6$ per group). (F) OVA class II tetramer staining of cells pooled from mechanically homogenized spleen and peripheral lymph nodes after OVA sensitization protocol as in $\mathbf{D}$. (G) Quantification of results in F. Mean and SEM together with individual data points are shown for $n=3(B \mathrm{Bl} / 6), n=6\left(E z h 2^{\mathrm{fl} / \mathrm{fI}}\right), n=5\left(E z h 2^{\mathrm{fl} / \mathrm{fl}} C d 4^{\mathrm{Cre}}\right)$. Statistical analysis by Kruskal-Wallis $H$ test with Dunn's post hoc test.

To examine Th2 development in mice lacking Ezh2 in T cells, we isolated splenocytes from $E z h 2^{\text {1// }}$ $C d 4^{c r e}$ mice and $E z h 2^{1 / 1 / l}$ control mice 10 days following initial sensitization to OVA. We then stimulated cytokine production with PMA/ionomycin for 5 hours in the presence of GolgiStop protein transport inhibitor for the final 2 hours prior to assessing intracellular IFN- $\gamma$ and IL- 4 cytokine accumulation by flow cytometry. We found that within the antigen-experienced $\mathrm{CD} 4^{+} \mathrm{T}$ cell compartment ( $\left.\mathrm{CD} 44^{+}\right)$, frequencies of IFN- $\gamma$ - and IL-4-producing cells did not differ between $E z h 2^{\mathrm{A} / \mathrm{ll}} C d 4^{\mathrm{Cre}}$ mice and $E z h 2^{\mathrm{n} / \mathrm{l}}$ control mice (Figure 3, D and E), suggesting that Th2 cells can develop in the absence of Ezh2.

Examination of the frequency of OVA-specific $\mathrm{CD} 4^{+} \mathrm{T}$ cells in the spleen and peripheral lymph nodes of $E z h 2^{\mathrm{A} / \mathrm{ll}} C d 4^{\mathrm{Cre}}$ and control floxed mice 10 days following initial OVA sensitization revealed a dramatic reduction in the number of OVA-specific $C D 4^{+} \mathrm{T}$ cells in $E z h 2^{\mathrm{a} / \mathrm{fl}} C d 4^{\mathrm{Cre}}$ mice compared with $E z h 2^{\mathrm{A} / \mathrm{fl}}$ mice (Figure 3, $\mathrm{F}$ and $\mathrm{G}$ ). Indeed, the frequency of tetramer ${ }^{+} \mathrm{CD} 4^{+} \mathrm{T}$ cells from $E z h 2^{\mathrm{n} / \mathrm{fl}}$ $C d 4^{c r e}$ mice was statistically indistinguishable from background levels of nonsensitized C57BL/6 controls (Figure 3, F and G). These data, together with the lack of OVA-specific IgE, suggest that Ezh2 is required to generate antigen-specific memory.

We hypothesized that the failure to generate antigen-specific $\mathrm{CD} 4^{+} \mathrm{T}$ cells in the absence of Ezh2 was due to a defect in $\mathrm{T}$ cell proliferation. Surprisingly, when we labeled $\mathrm{CD} 4^{+} \mathrm{T}$ cells from $E z h 2^{1 / 1 \mathrm{ll}} C d 4^{\text {cre }}$ and C57BL/6 control mice with CellTrace Violet dye and activated them in vitro using anti-CD3 and antiCD28 antibodies, we saw similar degrees of progressive dye dilution in both $E z h 2^{\mathrm{B} / \mathrm{ll}} \mathrm{Cd} 4^{\mathrm{Cre}}$ and C57BL/6 control mice, as well as similar upregulation of the activation marker CD25 (Figure 4A), suggesting intact activation responses and normal cell division. However, when we measured cell numbers over time, we found a profound defect in clonal expansion of cells from $E z h 2^{2 / / 1 / 1} C d 4^{\text {Cre }}$ mice (Figure 4B). Quantitation of cell death processes using annexin $\mathrm{V}$ and propidium iodide staining following cell activation showed a large increase in the proportion of $E z h 2^{\mathrm{A} / \mathrm{ll}} \mathrm{Cd} 4^{\mathrm{Cre}}$ cells undergoing apoptosis compared with WT cells (Figure 4, $\mathrm{C}$ and $\mathrm{D}$ ). This premature death in cells lacking PRC2 function therefore prevents a robust $\mathrm{T}$ cell expansion, thereby curtailing inflammatory $\mathrm{CD} 4^{+} \mathrm{T}$ cell response.

Inhibition of Ezh2 suppresses T cell expansion and allergic responses in vivo. Having shown that mice deficient in PRC2 components do not develop allergic inflammation, we used the Ezh2 small-molecule inhibitor GSK126 to test its therapeutic potential for allergic disease. GSK126 is a selective, $S$-adenosyl-L-methionine-competitive small-molecule inhibitor of Ezh2 methyltransferase activity that has been shown to suppress the growth of tumors in mouse models (17). Given that activation of T cells lacking Ezh2 function resulted in induction of apoptosis and a diminished immune response, we hypothesized that small-molecule inhibition of Ezh2 will similarly prevent $\mathrm{T}$ cell expansion. To test this, we activated WT CD4 ${ }^{+} \mathrm{T}$ cells in vitro using anti-CD3 and anti-CD28 antibodies, and exposed them to GSK126 for 3 days. As expected, we observed a dose-dependent reduction in the number of cells recovered in the culture compared with vehicle control (Figure 5A), with $10 \mu \mathrm{M}$ GSK126 sufficient to cause complete cell death at this time point. Importantly, H3K27me3 levels were significantly reduced by this dose of GSK126 at the earlier time point of 44 hours (Figure 5B), demonstrating on-target effects on Ezh2.

We then inhibited Ezh2 activity in vivo by administering GSK126 $(75-150 \mathrm{mg} / \mathrm{kg})$ by oral gavage on each day of OVA aerosol challenge, 4 hours after the OVA exposure (Figure 5C). Prior experiments using control (non-OVA-exposed) C57BL/ 6 mice found these doses of GSK126 to be well tolerated and to not affect BAL cellularity or cause any noticeable effects (Supplemental Figure 7). Consistent with 
A
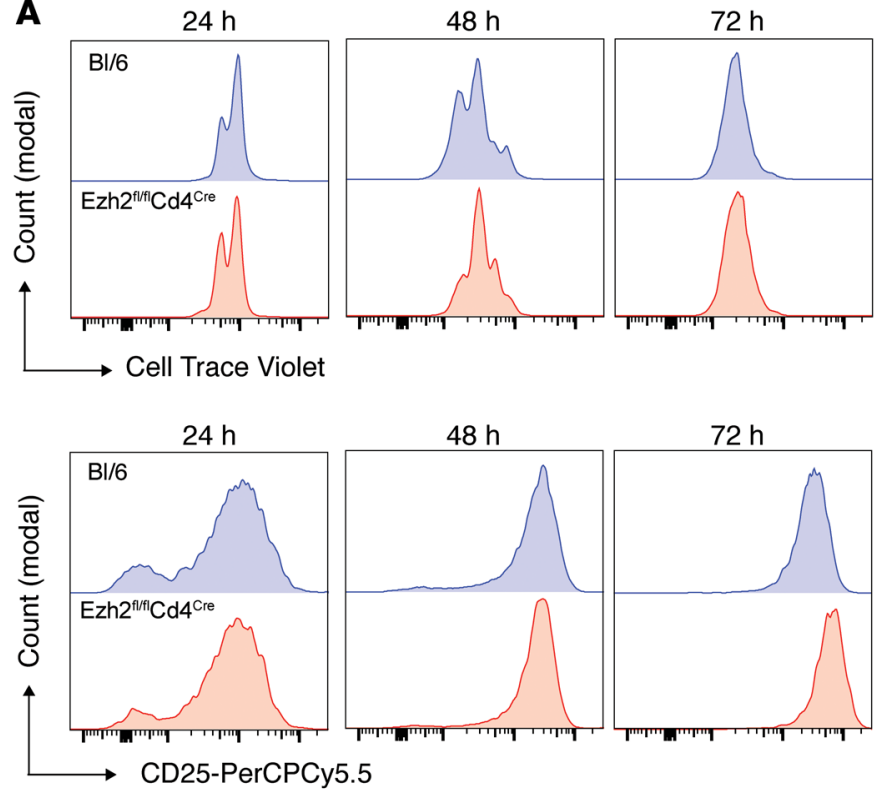

\section{$\mathbf{C}_{B / / 6}$}

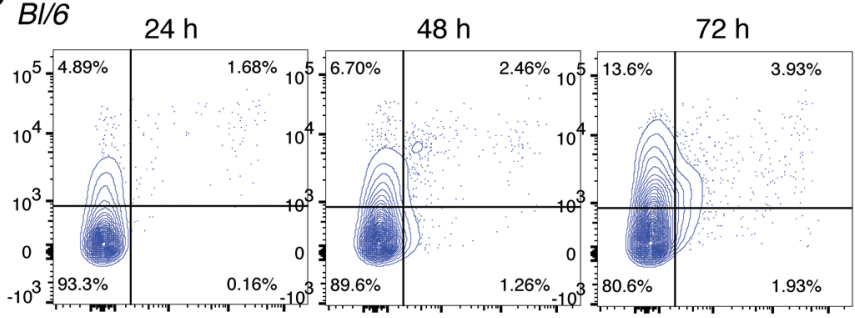

Ezh2 $2^{\text {lift }} \mathrm{C} d 4^{\text {Cre }}$
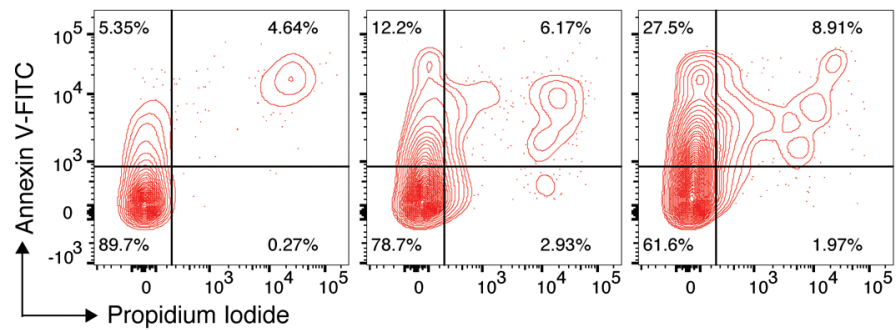

B
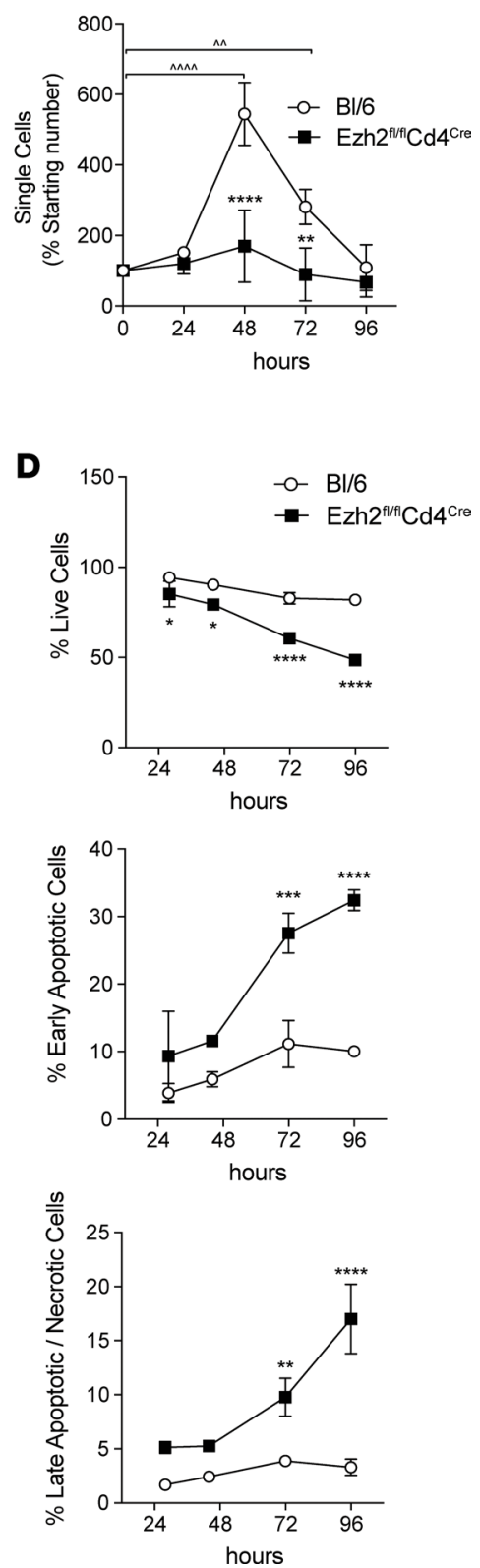

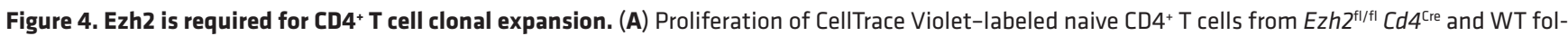
lowing anti-CD3 and anti-CD28 activation in vitro. Upregulation of the activation marker CD25 is displayed in the bottom panel. (B) Cell numbers recovered from Ezh $2^{\mathrm{fl} / \mathrm{fl}} \mathrm{Cd} 4^{\mathrm{Cre}}$ and WT cultures from A. Mean and SEM are shown $(n=3)$. Statistical analysis by 2-way ANOVA with Bonferroni's post hoc test. (C) Annexin $V$ and propidium iodide staining of cells activated as in $\mathbf{A}$. Live cells (bottom-left quadrant), early apoptotic cells (top-left quadrant), and late apoptotic/necrotic cells (top-right quadrant). (D) Quantification of results in C. Group sizes and statistical analysis are as in $\mathbf{B}$. ${ }^{*} P<0.05,{ }^{* *} P<0.01,{ }^{* * *} P<$ $0.001,{ }^{* * * *} P<0.0001$ compared with $C 57 \mathrm{BL} / 6(\mathrm{BI} / 6)$ control at that time point. ${ }^{\wedge} P P<0.01,{ }^{\wedge \wedge \wedge} P<0.0001$ compared with the 0 -hour time point.

previous experiments, OVA challenge of vehicle-treated control mice resulted in the accumulation of predominantly eosinophils in the airspace, along with smaller numbers of neutrophils and T cells (Figure 5D). The $150-\mathrm{mg} / \mathrm{kg}$ dose of GSK126 resulted in a dramatic reduction in each of these individual populations as well as total BAL leukocytes (Figure 5D). $\mathrm{PAS}^{+}$mucous-producing epithelial cells and levels of inflammation in the lung tissue were similarly reduced in the airways of mice treated with 150 $\mathrm{mg} / \mathrm{kg}$ GSK126 (Figure 5, E and F). The $75-\mathrm{mg} / \mathrm{kg}$ dose of GSK126 had no effect on BAL cells (Figure 5D), PAS staining (data not shown), or lung tissue inflammation (data not shown) compared with vehicle-treated mice. The administration of this drug did not lead to a global loss of immune cells, as the numbers of lymphocytes and granulocytes in the spleen were unchanged (Supplemental Figure 8). 
A
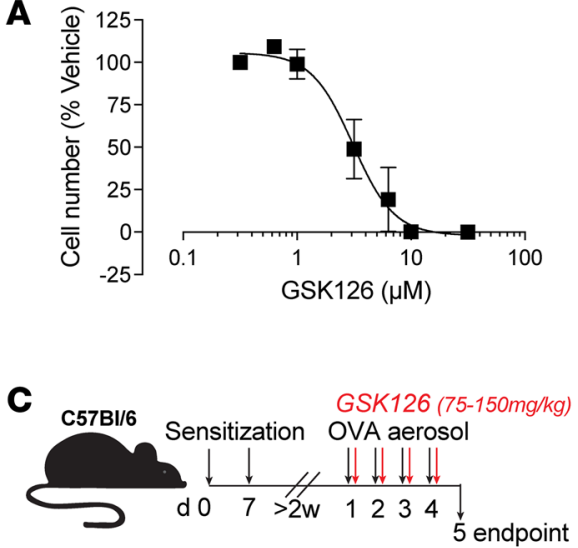

E

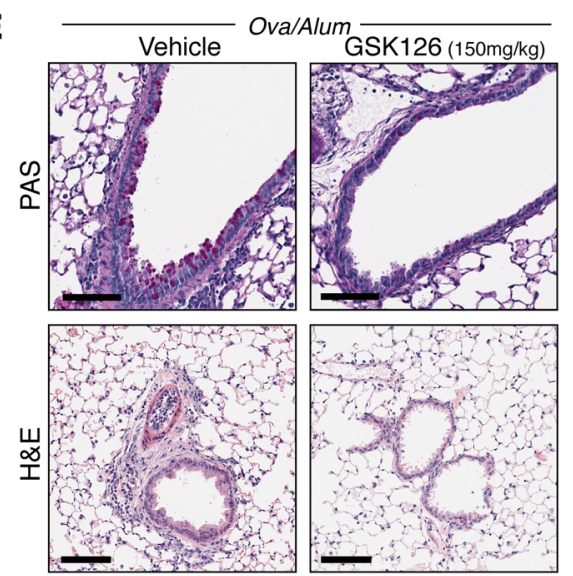

F
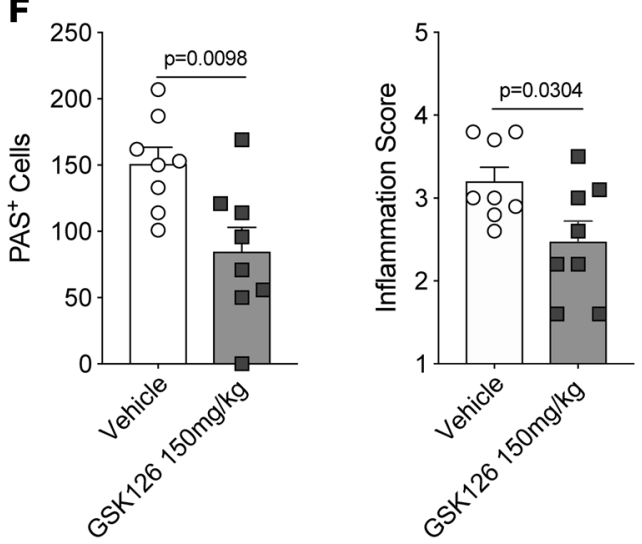

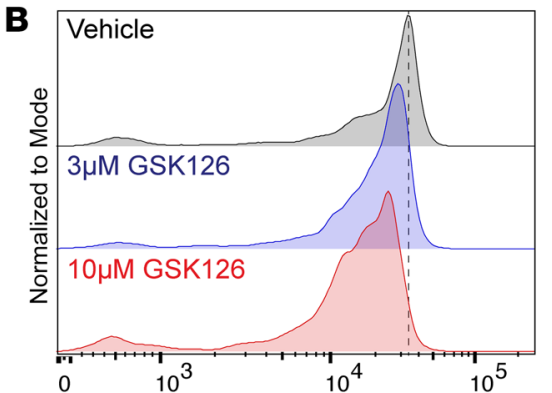

H3K27me3-A647
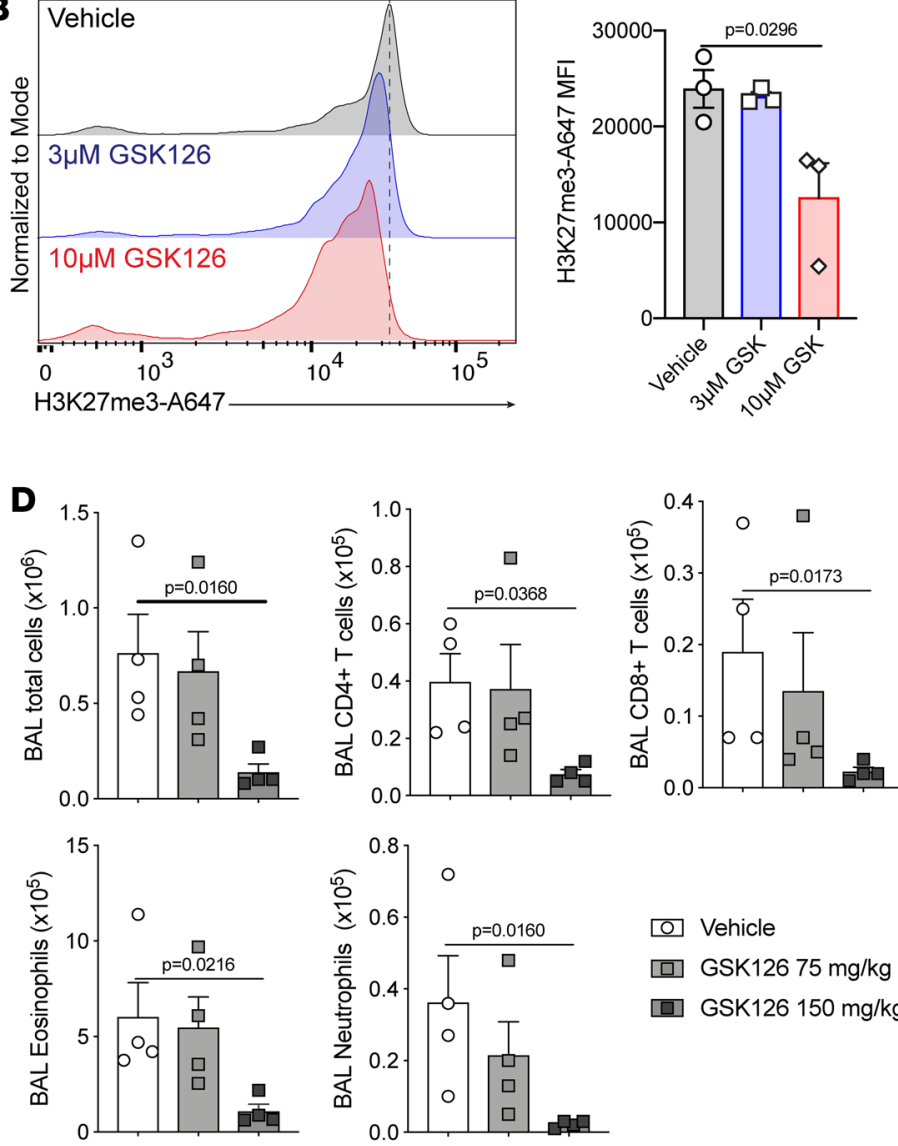

0 Vehicle

[ᄆ GSK126 $75 \mathrm{mg} / \mathrm{kg}$

마 GSK126 $150 \mathrm{mg} / \mathrm{kg}$
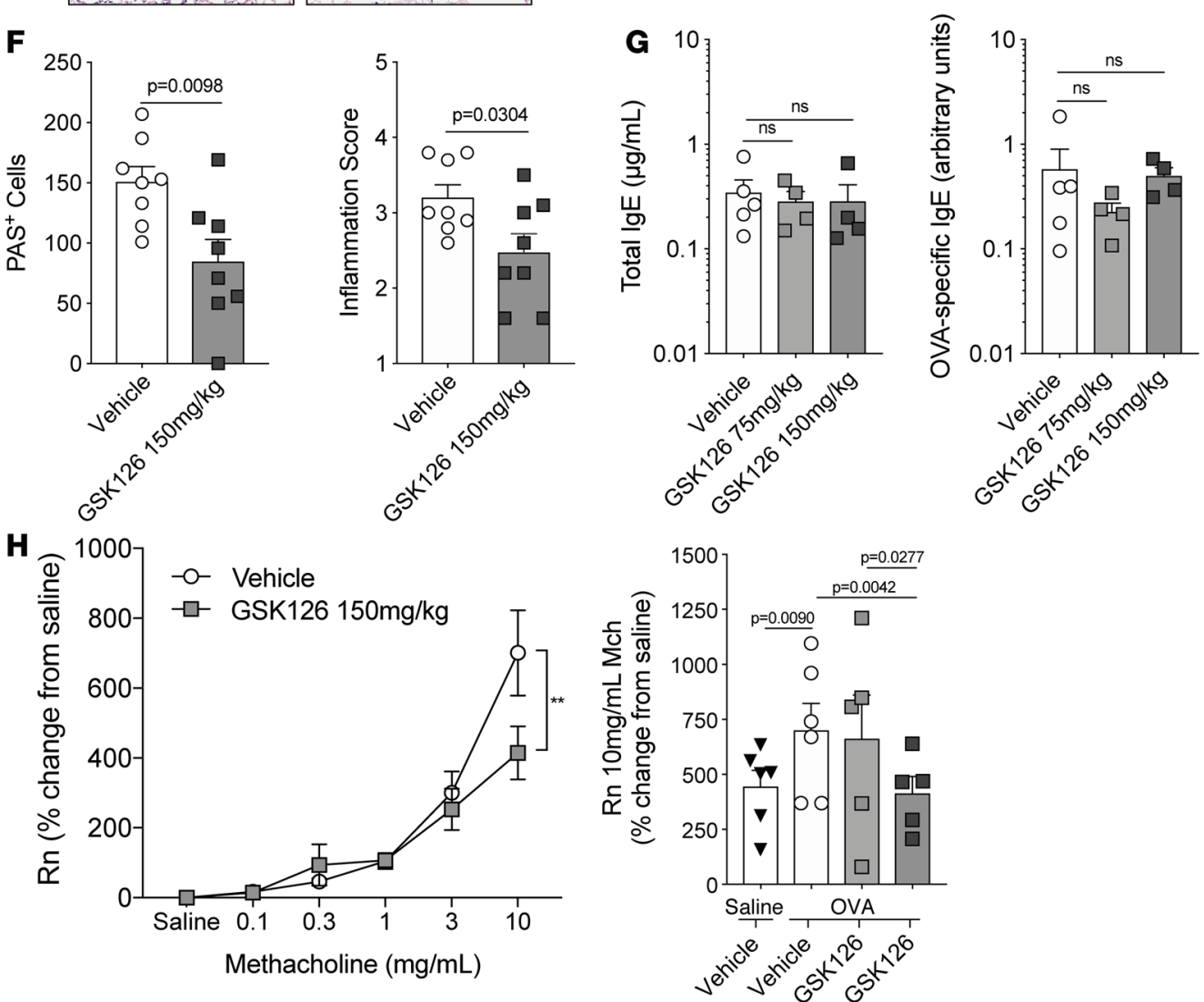

(18) $1(0)$

100 
Figure 5. Small-molecule inhibition of Ezh2 suppresses the development of allergic inflammation. (A) Effect of Ezh2 inhibitor GSK126 on survival of activated C57BL/ 6 mouse CD4+ $T$ cells after 3 days in vitro. Data are shown as the mean percentage of the vehicle control cell number \pm SEM from $3-5$ measurements at each concentration of GSK126. (B) H3K27me3 levels in activated C57BL/6 mouse CD4+ $T$ cells (as in A) after 44 hours. Representative histograms are shown together with median fluorescence intensity (MFI) from $n=3$ mice. Data were analyzed by 2-way ANOVA with Holm-Sidak post hoc test. (C) Experimental protocol of GSK126 administration by oral gavage in OVA-induced allergic inflammation. (D) Quantification of flow cytometric analysis of bronchoalveolar lavage infiltrate following GSK126 administration in OVA model of allergic inflammation in C57BL/6 mice ( $n=4$ per group). Mean and SEM together with individual data points are shown. Statistical analysis by Kruskal-Wallis $H$ test with Dunn's post hoc test. (E) Representative histological analysis (representative of $n=8$ per group, pooled from 2 independent experiments) of the airways of mice treated with GSK126 or vehicle control in OVA model as per C. Scale bars: $100 \mu \mathrm{m}$. (F) Quantification of histological analysis showing the frequency of PAS cells and the inflammation score. Mean and SEM together with individual data points are shown for $n=8$ per group. Statistical significance was determined by Student's $t$ test. (G) OVA-specific IgE and total IgE detected in serum of mice following OVA challenge and GSK126 administration. Mean and SEM are shown for $n=5$ (vehicle), $n=4$ (each GSK dose). Statistical analysis by Kruskal-Wallis $H$ test with Dunn's post hoc test. (H) Airway resistance (Rn) following OVA-induced allergic inflammation in C57BL/6 mice treated with vehicle or GSK126. Full methacholine dose-response for vehicle- and CSK126-treated (150 mg/kg) groups is shown in the left panel and all groups' airway resistance to $10 \mathrm{mg} / \mathrm{ml}$ methacholine is shown in the right panel. Mean and SEM together with individual data points are shown for $n=6$ (saline/vehicle), $n=6$ (OVA/vehicle), $n=5$ (OVA/75 mg/kg GSK126), $n=5$ (OVA/150 mg/kg GSK126). Data were analyzed by 2-way ANOVA with Bonferroni's post hoc test. ${ }^{* *} P<0.01$ with specific comparisons denoted in right-hand panel.

Similarly, GSK126 administration did not alter H3K27me3 levels in these splenic populations (Supplemental Figure 9). The concentration of OVA-specific IgE and total $\operatorname{IgE}$ was also unchanged in the serum of mice treated with GSK126 (Figure 5F), suggesting that inhibition of Ezh2 is a viable therapeutic strategy despite existing humoral and cellular memory in allergic individuals. Importantly, the $150-\mathrm{mg} / \mathrm{kg}$ dose of GSK126 also provided complete protection against OVA-induced airway hyperresponsiveness (Figure $5 \mathrm{H}$ ).

Inhibition of Ezh2 suppresses established inflammation. In order to further test the therapeutic potential of Ezh2 inhibition in established inflammation, we performed a single-hit experiment whereby allergic inflammation was established in our standard OVA model of systemic sensitization followed by daily nebulized OVA exposure for 4 days. On the fifth day, rather than assessing allergic inflammation as we had previously done, we administered a single dose of GSK126 $(150 \mathrm{mg} / \mathrm{kg})$ by oral gavage and then assessed bronchoalveolar inflammation 3 days later (Supplemental Figure 10A). This single dose given following established inflammation resulted in reduced BAL $\mathrm{CD} 4^{+} \mathrm{T}$ cell numbers and did not alter granulocyte numbers, suggesting a selective effect on actively dividing, allergy-promoting $\mathrm{T}$ cells in the lung microenvironment (Supplemental Figure 10B).

We then extended the therapeutic regimen to 3 doses (day 5, 8, and 10) after establishing inflammation by OVA challenge on days 1-4, and then assessed airway inflammation on day 11 (Figure 6A). Inhibition of Ezh2 in this therapeutic regimen resulted in reduced airway inflammation (Figure 6B), with total BAL cells, $\mathrm{CD} 4^{+} \mathrm{T}$ cells, eosinophils, and B cells all significantly reduced following GSK126 treatment (Figure 6, B and C). Interestingly, numbers of BAL eosinophils recovered were highly correlated with numbers of BAL CD4 $4^{+} \mathrm{T}$ cells (Figure $6 \mathrm{D}$; Pearson's $\mathrm{R}^{2}=0.9261, P<0.0001$ ). Examination of lung histopathology revealed reduced PAS $^{+}$mucous-producing cells following therapeutic GSK126 treatment (Figure 6, E and F). However, lung inflammation levels had ablated by this point (7 days after final OVA challenge) and all groups were statistically indistinguishable (Figure 6, E and F). Overall, these data validate our gene inactivation experiments and show that inhibiting the enzymatic activity of Ezh2 is a feasible strategy for suppressing allergic responses, even in established inflammation.

\section{Discussion}

Previously, we implicated the Suv39h/H3K9me3/HP1 pathway in Th2 cell stability by focusing specifically on Suv39h1 (10). In this study, we took a broader approach in an attempt to identify which other epigenetic silencing components were upregulated after human $\mathrm{CD} 4^{+} \mathrm{T}$ cell activation. Surprisingly, the genes that were upregulated after activation were almost all from the PRC2/H3K27me3 and Suv39h/H3K9me3/ HP1 pathways. Both of these pathways are critical to the formation of repressive chromatin. This suggests that establishment of gene silencing and heterochromatin formation or maintenance is an important part of the first stages of a lymphocyte response.

Because of the role of Suv39h1 in Th2 cell stability it was logical to think that the other components of this pathway may also play an important role in the development of allergy. In this pathway, methylation of H3K9 is imparted not only by Suv39h1 but also G9a, GLP, SETDB1, and Suv39h2 enzymes. However, the enzymes responsible for $\mathrm{H} 3 \mathrm{~K} 9$ trimethylation (H3K9me3) are predominantly Suv39h1 
A Therapeutic regimen
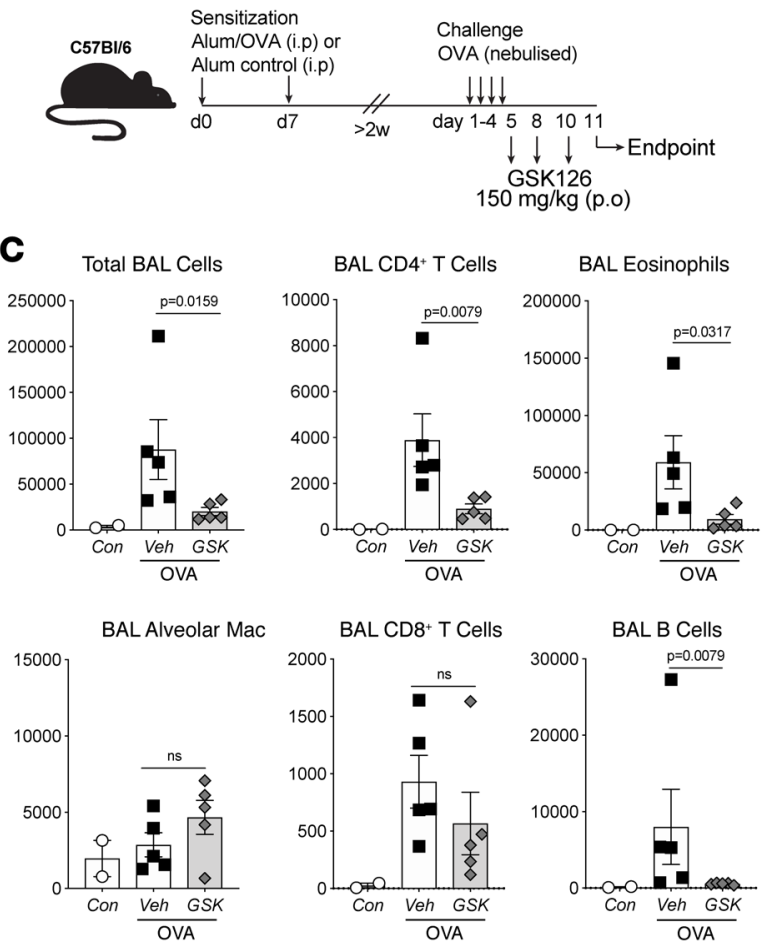

$\mathbf{E}$

D

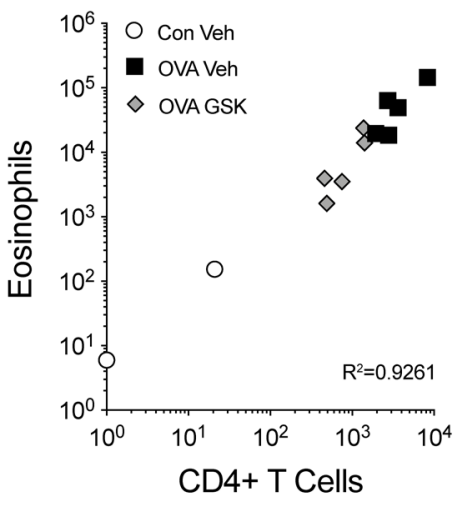

B
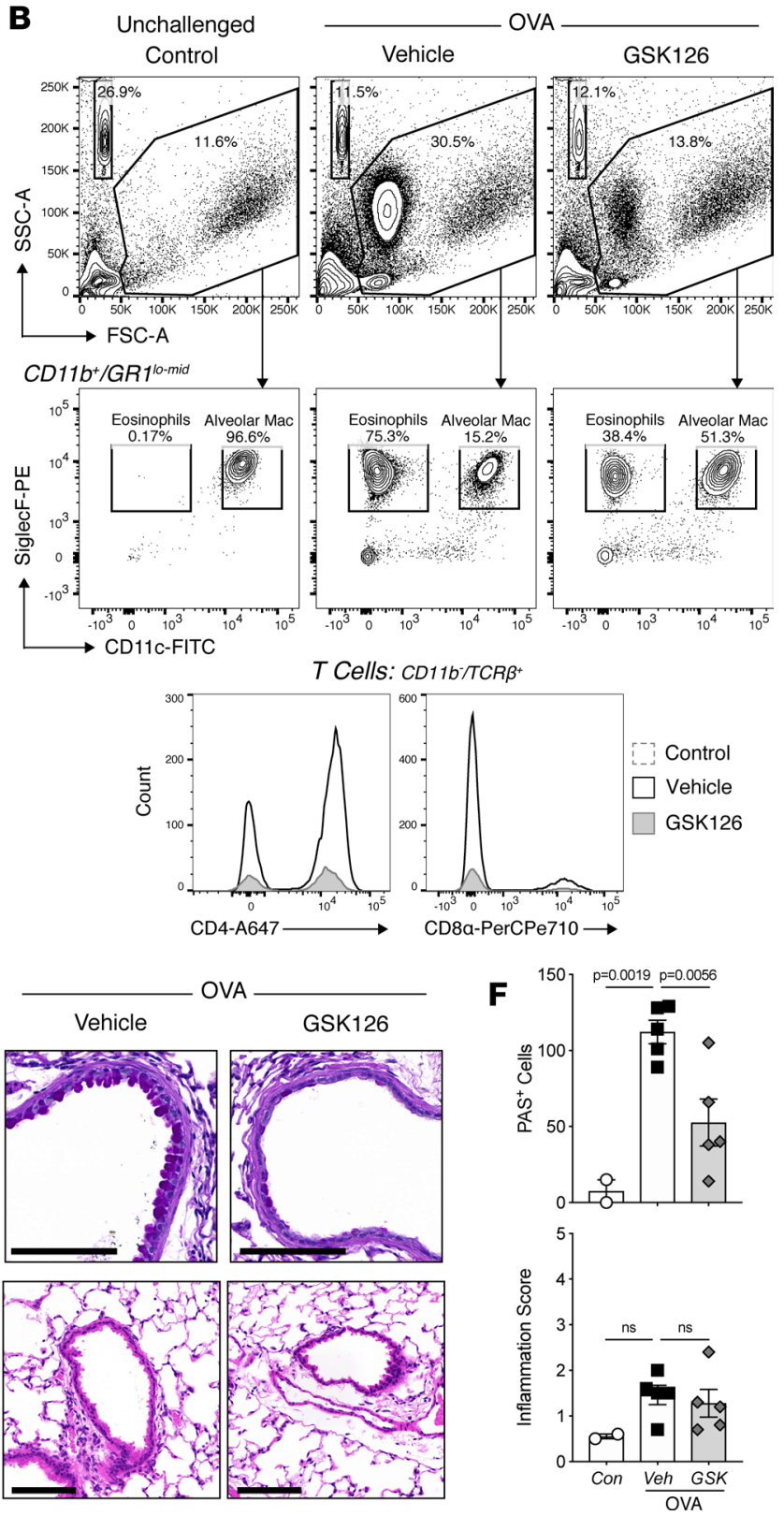

Figure 6. Inhibition of Ezh2 can treat established allergic inflammation. (A) Experimental protocol using Ezh2 inhibitor GSK126 in established inflammation. (B) Representative flow cytometry plots of bronchoalveolar lavage (BAL) samples from GSK126-treated (150 mg/kg by oral gavage) and vehicle-treated C57BL/6 mice. Data shown are representative of $n=5$ OVA/vehicle (Veh) and OVA/CSK groups, $n=2$ unchallenged controls (Con) shown for reference purposes. (C) Quantification of results in B. Mean and SEM together with individual data points are shown. Group sizes are as in B. Statistical analysis by Mann-Whitney $U$ test comparing vehicle and GSK126 groups. (D) Correlation of BAL eosinophils with BAL CD4 ${ }^{+} T$ cells in groups as per B. Individual data points are shown. (E) Representative histological analysis of the airways of mice following GSK126 or vehicle treatment in established inflammation. Scale bars: $100 \mu \mathrm{m}$. (F) Quantification of PAS ${ }^{+}$cells and inflammation score. Mean and SEM together with individual data points are shown. Group sizes are as in B. Statistical analysis by 1-way ANOVA with Holm-Sidak post hoc test.

and Suv39h2. H3K9me3 then serves as a binding site for a corepressor family, heterochromatin protein 1 (HP1 $\alpha,-\beta$, and $-\gamma)(18-20)$, which sterically inhibits the binding of transcriptional machinery and attracts diverse chromatin modifiers such as DNA methyltransferases (21) and Trim28 (22), allowing maintenance of silencing. Interestingly, we did not find a role for any of the cofactors we examined. It is possible that more than one HP1 molecule would need to be inactivated in order to alter T cell-driven inflammation. T cell-specific TIF1 $\beta$ deficiency has previously been shown to result in increased TGF- $\beta$ production and spontaneous Th17 and T regulatory cell development (23). However, despite these alterations, TIF1 $\beta$ deficiency did not enhance or diminish the allergic response. 
In contrast, the PRC2 pathway was essential for T cells to drive the development of allergic inflammation. In this pathway, trimethylation of H3K27 is mediated by PRC2, which consists of Ezh2, Eed, Suz12, and accessory factors such as Jarid2 and RBBP4 and -7. Ezh2 (and the related Ezh1) provide the enzymatic component of PRC2, while Eed and Suz12 are essential for complex stability (24). Ezh2 plays an important role in both $\mathrm{B}$ and $\mathrm{T}$ cell development (reviewed in ref. 25) and was recently implicated in allergic asthma development, as Ezh2-deficient T cells were shown to enhance asthma pathology after adoptive transfer of in vitro-activated T cells (26). Our findings clearly contrast with the conclusions from this report. Tumes et al. used a Th2 in vitro polarization of OVA-specific T cell receptor-transgenic Ezh2-deficient CD4 ${ }^{+} \mathrm{T}$ cells prior to adoptive transfer into OVA-sensitized mice (26). In contrast, here we directly tested the importance of Ezh2 to the generation of T cell responses in vivo. As shown in Figure 2, we found a profound deficiency in such responses and these results were phenocopied by the conditional inactivation of the nonredundant PRC2 components, Suz12 and Eed. We speculate that the increased proliferative drive from the OVA-specific transgenic model may overcome the proliferative defect observed in our studies which used the endogenous $\mathrm{T}$ cell response. Our results showing defective T cell responses in the absence of EZH2 are also concordant with studies showing defective Th1 response to Listeria monocytogenes (27) and Toxoplasma gondii (28), as well as defective allogeneic graft-versus-host responses (29). Taken together, our data generated from independently inactivating 3 components of the PRC2 complex in T cells and small-molecule inhibition of Ezh2 demonstrate that PRC2 function is absolutely required for the manifestation of allergic responses. In this case, unlike our previous work on Suv39h1 inhibition, lack of allergic responses in the absence of PRC2 function does not appear to be due to skewing toward the Th1 phenotype, but rather due to initiation of apoptosis following cell activation.

Targeting the enzymes that are responsible for laying down epigenetic modifications to chromatin has recently become possible with the design of several inhibitory drugs $(30,31)$. Concerns that targeting part of the epigenetic machinery would have widespread deleterious effects have been alleviated by clinical studies demonstrating that selective small-molecule inhibitors that target individual epigenetic enzymes are well tolerated in cancer patients, including many that target EZH2 $(31,32)$. Indeed, we did not observe any systemic effects at the doses of GSK126 we have used, nor did we notice effects on the number of resident alveolar macrophages in the airways. One could speculate that individuals with inhibited PRC2 function could be immunocompromised when confronted with an immunological challenge, such as virus exposure. Our own data support a selective effect of Ezh2 inhibition on rapidly dividing cells, such as $\mathrm{CD} 4{ }^{+} \mathrm{T}$ cells mounting an allergic response, rather than terminally differentiated and relatively transcriptionally silent granulocyte populations. This effect may be due to global destabilization of H3K27me3-dependent heterochromatin, which may affect cell viability as the genome is placed in a unique position of stress through the process of rapid and massive clonal expansion (33). Alternatively, loss of PRC2 function may cause dysregulated transcription of key target genes such as Cdkn2a, a known target of Ezh2 in multiple cell types (34) that is involved in the stabilization of p53 regulating cell cycle progression (35), and furthermore has been shown to regulate lymphoid cell survival (36). These effects on dividing T cells might somewhat blunt adaptive responses; however, innate responses would likely remain intact. Furthermore, it is plausible that careful titration of EZH2 inhibition levels may be able to provide protection from unwanted allergic/autoimmune activation, while retaining sufficient $\mathrm{T}$ cell response when antigen load is high. We therefore consider that EZH2 inhibition represents a viable therapeutic strategy to target the lymphocytes that drive lung inflammation.

Our data using therapeutic regimens of GSK126 provide further evidence that Ezh2 inhibition is indeed a viable therapeutic strategy for diseases such as allergic asthma. While genetic deletion of Ezh2 prevented the development of antigen-specific $\mathrm{CD}^{+} \mathrm{T}$ cells and antigen-specific IgE (albeit only directly shown in the OVA model), thereby precluding a subsequent immune-response antigen challenge, GSK126 treatment was able to reduce airway inflammation and hyperresponsiveness even when antigen-specific memory was well established. Indeed, therapeutic efficacy of Ezh2 inhibition could be seen when this drug was administered in the context of ongoing established inflammation. Interestingly, our observed correlation between airway eosinophilia and $\mathrm{CD}^{+} \mathrm{T}$ cell numbers reflects what is seen in human asthmatic disease (37) and importantly also correlates with asthma severity (38). These results provide a rationale to expect utility of an EZH2 inhibitor as a therapeutic in uncontrolled disease, and not just as a prophylactic treatment.

Given the tolerability of EZH2 inhibitors in human studies, even when dosed through systemic routes, the transition from oncology to treatments for inflammation could be expected to be smooth and rapid. This is especially true given that the dosing regimen used for the treatment of allergic inflamma- 
tion would be expected to be much more restricted than that for cancer, and ideally organ restricted (i.e., to the lungs for allergic asthma). The most advanced of these studies involve tazemetostat (Epizyme Inc), that has successfully completed phase I and entered phase II clinical trials. Other early-phase trials have been initiated by GSK (NCT02082977), Constellation Pharmaceuticals (CPI-2105, NCT02395601), and Novartis (MAK683, NCT02900651). These first-in-human studies will provide important insights into the potential of the various Ezh2 inhibitors for the treatment of allergic inflammation. Finally, we believe that the suppression of inflammation associated with EZH2 inhibition could also be used to treat other conditions in which activated $\mathrm{T}$ or $\mathrm{B}$ lymphocytes drive the inflammation. As the inhibitors have already undergone safety and efficacy trials, we would be able to more rapidly translate this approach into clinically testing such possibilities.

\section{Methods}

Mice

$E z h 2^{\mathrm{f} / \mathrm{fl}}(12), E e d^{\mathrm{fl} / \mathrm{fl}}(16), \operatorname{Suz} 2^{\mathrm{fl} / \mathrm{fl}}(15), \operatorname{Cbx} 5^{\mathrm{n} / \mathrm{fl}}(10)$, and Trim2 $8^{\mathrm{fl} / \mathrm{fl}}$ (39) mice were described previously. $\mathrm{Cb} x \mathrm{I}^{\mathrm{fl} / \mathrm{fl}}$ mice were a gift from Florence Cammas (Institute of Genetics and Molecular and Cellular Biology, Strasbourg, France). The floxed strains were crossed with $C d 4^{\text {cre }}$ mice (13). All mice lines have been maintained on a C57BL/6 (Ly5.2) background and were used between 6 and 12 weeks of age and were age and sex matched.

\section{OVA model of allergic lung inflammation}

Mice were immunized with an intraperitoneal injection of $200 \mu 1$ alum/OVA containing $20 \mu \mathrm{g}$ low-endotoxin OVA (Worthington) and $2.25 \mathrm{mg}$ aluminium hydroxide (Sigma-Aldrich) in sterile PBS on day 0 and day 7. Mice were then rested for a minimum of 2 weeks and then challenged daily for 4 days with nebulized $2 \%$ (w/v) OVA (Sigma-Aldrich) in PBS for 15 minutes. The day after the final challenge (or at a time point described in the text), mice were sacrificed and BAL (250 $\mu 1$ twice with sterile PBS) was performed, from which cellular infiltrate was analyzed by flow cytometry and acellular BAL fluid was analyzed by Bio-Plex Pro cytokine assay (Bio-Rad). Lung tissue was then fixed by inserting $1 \mathrm{ml}$ of $10 \%$ formalin via the trachea into the lungs and leaving it to set in situ for at least 30 minutes. The midsection of the left lobe was then excised and placed in liquid formalin for a further 24 hours prior to paraffin embedding, sectioning, and staining with periodic acid-Schiff (PAS) and hematoxylin and eosin (H\&E) stains. Stained slides were imaged using an Aperio Digital Pathology Slide Scanner and quantitated by counting PAS-positive epithelial cells and scoring leukocyte infiltration in $\mathrm{H} \& \mathrm{E}$-stained sections according to the scoring chart in Supplemental Figure 11. Scores from at least five $\times 20$ magnification fields of each stain were averaged for each mouse.

\section{Respiratory mechanics}

Lung function was assessed by the forced oscillation technique using a FlexiVent system with FX1 module (SCIREQ). Mice were anesthetized with ketamine $(150 \mathrm{mg} / \mathrm{kg})$ and xylazine $(15 \mathrm{mg} / \mathrm{kg})$ and then cannulated by tracheostomy with ligation. Baseline respiratory mechanics were recorded, followed by aerosolized saline and then increasing doses of methacholine $(\mathrm{MCh})(0.1-30 \mathrm{mg} / \mathrm{ml})$. Respiratory impedance $\left(\mathrm{Z}_{\mathrm{rs}}\right)$ was measured and partitioned into airway and parenchymal components through fitting to the Constant Phase Model from which Newtonian resistance $\left(\mathrm{R}_{\mathrm{n}}\right.$; equivalent to airway resistance) was calculated.

\section{HDM extract model of allergic asthma}

Mice were challenged with intranasal administration of protein extract from whole HDMs (Dermatophagoides pteronyssinus, Greer Laboratories Inc.) on days 0-2 (10 $\mu \mathrm{g} / \mathrm{mouse})$ and then on days $14-17$ (1 $\mu \mathrm{g} / \mathrm{mouse})$ and then sacrificed on day 18. These HDM extract doses of $10 \mu \mathrm{g}$ and $1 \mu \mathrm{g}$ contained $1.16 \mathrm{EU}$ and 0.116 EU of endotoxin (LPS), respectively. Endpoints were the same as for the OVA model above.

\section{GSK126 in vivo administration}

GSK126 (Xcessbio Biosciences) was administered by oral gavage at doses of $75 \mathrm{mg} / \mathrm{kg}$ and $150 \mathrm{mg} / \mathrm{kg}$ in $20 \%$ Captisol vehicle. 


\section{ELISAS}

Total IgE and OVA-specific IgE were measured by enzyme-linked immunosorbent assay (ELISA). Briefly, 96-well plates were coated with either rat anti-mouse IgE (to measure total IgE; Southern Biotech clone 23G2, catalog 1130-01) or OVA protein (for OVA-specific IgE) in PBS overnight. Samples were diluted (total IgE 1:50-1:200 and OVA-specific IgE 1:50-1:100 based on pilot assays), and incubated in duplicate, prior to incubation with goat anti-mouse Fc-specific polyclonal anti-IgE-HRP and colorimetric detection using TMB substrate (Thermo Fisher Scientific, 34028), measuring absorbance at $450 \mathrm{~nm}$. Absorbance values were converted to total IgE levels by reference to a mouse anti-dinitrophenyl IgE (clone SPE-7) standard curve $(0.1 \mathrm{ng} / \mathrm{ml}$ to $1 \mu \mathrm{g} / \mathrm{ml})$ that was included on the same plate. OVA-specific IgE absorbance was expressed relative to a reference OVA-immunized WT mouse that was selected prior to measurement. All ELISAs were performed blinded to treatment and genotype.

\section{T cell cultures}

Naive CD4 ${ }^{+} \mathrm{T}$ cells were isolated from a single-cell suspension from mouse spleen by a MACS isolation kit (Miltenyi Biotec). Cells were labelled with CellTrace Violet (Thermo Fisher Scientific) and then activated in 96-well plates precoated with anti-CD3 $(10 \mu \mathrm{g} / \mathrm{ml})$ and anti-CD28 $(5 \mu \mathrm{g} / \mathrm{ml})$ in RPMI-1640 medium supplemented with $10 \%$ heat-inactivated fetal bovine serum, $2 \mathrm{mM}$ GlutaMAX, and $0.05 \mathrm{mM} \beta$-mercaptoethanol. Where indicated, cells were treated with the Ezh2 inhibitor GSK126 at the onset of cell activation.

\section{Immunoblotting}

For immunoblotting, whole-cell extracts were prepared by lysing cells in RIPA buffer (Millipore). Bradford assay was performed to quantify total protein content in each lysate and samples of equivalent protein content were resolved in denaturing conditions in 4\%-12\% gradient SDS-PAGE (Life Technologies) and were transferred onto nitrocellulose membranes (Bio-Rad). Membranes were probed with the following antibodies: goat anti-actin-HRP (sc-1616, Santa Cruz); rabbit anti-Ezh2 (12408, Cell Signaling); rabbit anti-Suz12 (3737, Cell Signaling); mouse anti-HP1 $\alpha$ (05-689, Millipore); mouse anti-HP1 $\beta$ (MAB3448, Millipore); rabbit anti-laminB1 (ab16048, Abcam); mouse anti-TIF1 (MAB3662, Millipore); rabbit anti-H3K9me3 (ab8898, Abcam); and rabbit anti-H3K27me3 (07-449, Millipore).

\section{Flow cytometry}

The following staining panels of fluorochrome-conjugated antibodies against mouse antigens were used for analysis and sorting by flow cytometry.

BAL and spleen immunophenotyping panel. CD19 (1D3)-BUV395 and SiglecF (E50-2440)-PE were from BD Pharmingen. CD11c (N418)-FITC, CD8 $\alpha$ (53-6.7)-PerCPe710, Ly6c (HK1.4)-e450, GR1 (RB68C5)-PECy7, and TCR $\beta$ (H57-597)-APCe780 were from eBioscience. CD4 (GK1.5)-Alexa Fluor 647 and CD11b(M1/70)-Alexa Fluor 700 were generated internally.

Intracellular cytokine analysis. IL-4 (11B11)-BV421, CD4 (GK1.5)-PECy7, and CD44 (IM7)-APCCy7 were from Biolegend. IFN- $\gamma$ (XMG1.2)-APC and TCR $\beta$ (H57-597)-PE were from BD Pharmingen. B220 (RA3-6B2)-FITC was generated internally.

OVA tetramer panel. CD4 (GK1.5)-PECy7 and CD19 (6D5)-PerCPCy5.5 or B220 (RA3-6B2)-Pacific Blue were from Biolegend. CD44 (IM7)-FITC was from BD Pharmingen. TCR $\beta$ (H57-597)-APCe780 was from eBioscience. CD11b (M1/70)-AlexaFluor700 was generated internally.

T cell activation panel. CD25 (PC61.5)-PerCPCy5.5 was from eBioscience. CD69 (H1.2F3)-APC was from Miltenyi Biotec.

$C D 4^{+} T$ cell and B cell sorting panel. TCR $\beta$ (H57-597)-APC was from eBioscience. CD4 (GK1.5)-PE, CD8 $\alpha$ (53-6.7)-FITC, and CD19 (1D3)-Pacific Blue were generated internally.

H3K27me3 staining. Tri-methyl-histone H3 (K27) (C36B11)-Alexa Fluor 647 mAb was from Cell Signaling.

Surface staining was carried out at $4^{\circ} \mathrm{C}$ for 30 minutes and propidium iodide or SYTOX Blue (Thermo Fisher Scientific) exclusion was used as live cell indicator.

Intracellular cytokine and H3K27me3 staining was performed using a FoxP3/Transcription Factor Staining Buffer Set (Affymetrix eBioscience, 00-5523) following the manufacturer's instructions. PMA (20 $\mathrm{ng} / \mathrm{ml})$ and ionomycin $(500 \mathrm{ng} / \mathrm{ml})$ stimulation for 5 hours was used to stimulate cytokine production and BD GolgiStop Protein Transport Inhibitor (BD Pharmingen, 554724) was added for the final 2 hours to allow intracellular cytokine accumulation. 
Tetramer-based enrichment of OVA-specific $\mathrm{CD}^{+} \mathrm{T}$ cells was performed using PE-conjugated OVA2C and OVA-3C Peptide:MHC class II tetramers provided by James Moon (Massachusetts General Hospital, Boston, Massachusetts, USA), as previously described (40).

Annexin V-FITC (BD Pharmingen, 556419) staining was performed in $1 \times$ binding buffer $(10 \mathrm{mM}$ HEPES, $140 \mathrm{mM} \mathrm{NaCl}, 2.5 \mathrm{mM} \mathrm{CaCl}_{2}$ in PBS) prior to propidium iodide addition and analysis.

In all experiments, stained cells were analyzed using a BD FACSCanto II or a BD LSRFortessa X-20 using SPHERO Rainbow Calibration Beads to calculate absolute cell counts. Where indicated, cells were sorted using a BD FACSARIA III.

\section{Bioinformatic analysis and statistics}

The human naive $\mathrm{CD} 4^{+} \mathrm{T}$ cell activation microarray data set originally produced by Martínez-Llordella et al. (11) is publically available through the NCBI's Gene Expression Omnibus (GEO), accession GSE39594. These data were produced using the Affymetrix Human Genome U133 Plus 2.0 Array platform. These data are comprised of $\mathrm{CD}^{+} \mathrm{T}$ cells that have been cultured under different conditions - nonstimulated and anti-CD3 plus anti-CD28 for 24 hours. Each group contained 3 replicate samples giving a total of 6 samples. All CEL files were downloaded and probe expression was calculated and normalized using robust multi-array average expression (RMA) (41). Probes were filtered from the data if their expression was less than 6 in at least 3 samples. Probes with no Entrez gene ID were also removed. Analysis of these data was performed using the limma (42) software package. Differential expression was evaluated between groups relative to a fold-change threshold of 1.2 using linear models and empirical Bayes moderated t-statistics with a trended prior variance (43). $P$ values were adjusted using the Benjamini and Hochberg method to control the FDR below 5\%. The mean-difference plot was produced using limma's plotMD function.

All other statistical analysis throughout the manuscript was performed using GraphPad Prism 7 software. Student's $t$ tests (2 tailed), 1-way ANOVA, or 2-way ANOVA were used or nonparametric equivalents as detailed in figure legends. $P$ values less than 0.05 were considered significant.

\section{Study approval}

Animal experiments were reviewed and approved by the Walter and Eliza Hall Institute Animal Ethics Committee, Parkville, Victoria, Australia.

\section{Author contributions}

SLN and RSA conceptualized the study. ALG provided formal analysis. CRK, NI, ACB, RYK, JCH, $\mathrm{PMH}$, and RSA conducted the investigations. CRK and RSA wrote the original draft of the manuscript. CRK, SLN, and RSA reviewed and edited the manuscript. CRK and ALG created the figures. SLN and RSA supervised the study and provided funding.

\section{Acknowledgments}

We thank Florence Cammas (IRCM, France) for the Cbx1, Cbx3, and Trim28 $8^{\mathrm{f} / \mathrm{fl}}$ mice, Alexander Tarakhovsky (Rockefeller University) for the $E z h 2^{\mathrm{fl} / \mathrm{fl}}$ mice, and Stuart Orkin (Dana-Farber Cancer Institute, Harvard) for the Eed ${ }^{\mathrm{l} / \mathrm{fl}}$ mice. We also thank James Moon (Massachusetts General Hospital) for providing class II OVA-MHC tetramers (OVA-2C, OVA-3C). Assessment of total and OVA-specific IgE was generously performed by Kristy O'Donnell in the laboratory of David Tarlinton (Monash University). This work was supported by grants and fellowships from the National Health and Medical Research Council of Australia (1125436 to CRK, 1100451 to RSA, and 1058238 and 1054925 to SLN), the Australian Research Council (130100541 to RSA), and an American Asthma Foundation Grant (to SLN and RSA). This study was made possible through Victorian State Government Operational Infrastructure Support and Australian Government NHMRC Independent Research Institute Infrastructure Support scheme and the Australian Cancer Research Fund.

Address correspondence to: Rhys S. Allan, Walter and Eliza Hall Institute of Medical Research, 1G Royal Parade, Parkville, Victoria, Australia 3052. Phone: 61.3.9345.2999; Email: rallan@wehi.edu.au. 
1. Gonzalo JA, et al. Eosinophil recruitment to the lung in a murine model of allergic inflammation. The role of T cells, chemokines, and adhesion receptors. J Clin Invest. 1996;98(10):2332-2345.

2. Lambrecht BN, Hammad H. Lung dendritic cells in respiratory viral infection and asthma: from protection to immunopathology. Annu Rev Immunol. 2012;30:243-270.

3. Murphy KM, Stockinger B. Effector T cell plasticity: flexibility in the face of changing circumstances. Nat Immunol. 2010;11(8):674-680

4. O'Shea JJ, Paul WE. Mechanisms underlying lineage commitment and plasticity of helper CD4+ T cells. Science. 2010;327(5969):1098-1102.

5. Allan RS, Nutt SL. Deciphering the epigenetic code of T lymphocytes. Immunol Rev. 2014;261(1):50-61.

6. Avni O, Lee D, Macian F, Szabo SJ, Glimcher LH, Rao A. T(H) cell differentiation is accompanied by dynamic changes in histone acetylation of cytokine genes. Nat Immunol. 2002;3(7):643-651.

7. Baguet A, Bix M. Chromatin landscape dynamics of the Il4-I113 locus during T helper 1 and 2 development. Proc Natl Acad Sci USA. 2004;101(31):11410-11415.

8. Grogan JL, et al. Basal chromatin modification at the IL-4 gene in helper T cells. J Immunol. 2003;171(12):6672-6679

9. Fields PE, Kim ST, Flavell RA. Cutting edge: changes in histone acetylation at the IL-4 and IFN-gamma loci accompany Th1/ Th2 differentiation. J Immunol. 2002;169(2):647-650.

10. Allan RS, et al. An epigenetic silencing pathway controlling T helper 2 cell lineage commitment. Nature. 2012;487(7406):249-253

11. Martínez-Llordella M, et al. CD28-inducible transcription factor DEC1 is required for efficient autoreactive $\mathrm{CD}^{+} \mathrm{T}$ cell response. J Exp Med. 2013;210(8):1603-1619.

12. $\mathrm{Su} \mathrm{IH}$, et al. Ezh2 controls B cell development through histone $\mathrm{H} 3$ methylation and Igh rearrangement. Nat Immunol. 2003;4(2):124-131.

13. Lee PP, et al. A critical role for Dnmt1 and DNA methylation in T cell development, function, and survival. Immunity. 2001;15(5):763-774.

14. Vasanthakumar A, et al. A non-canonical function of Ezh2 preserves immune homeostasis. EMBO Rep. 2017;18(4):619-631

15. Lee SC, et al. Polycomb repressive complex 2 component Suz12 is required for hematopoietic stem cell function and lymphopoiesis. Blood. 2015;126(2):167-175.

16. Xie H, et al. Polycomb repressive complex 2 regulates normal hematopoietic stem cell function in a developmental-stage-specific manner. Cell Stem Cell. 2014;14(1):68-80.

17. McCabe MT, et al. EZH2 inhibition as a therapeutic strategy for lymphoma with EZH2-activating mutations. Nature. 2012;492(7427):108-112.

18. Bannister AJ, et al. Selective recognition of methylated lysine 9 on histone H3 by the HP1 chromo domain. Nature. 2001;410(6824):120-124.

19. Elgin SC, Grewal SI. Heterochromatin: silence is golden. Curr Biol. 2003;13(23):R895-R898.

20. Lachner M, O'Carroll D, Rea S, Mechtler K, Jenuwein T. Methylation of histone H3 lysine 9 creates a binding site for HP1 proteins. Nature. 2001;410(6824):116-120.

21. Fuks F, Burgers WA, Brehm A, Hughes-Davies L, Kouzarides T. DNA methyltransferase Dnmt1 associates with histone deacetylase activity. Nat Genet. 2000;24(1):88-91.

22. Maison C, Almouzni G. HP1 and the dynamics of heterochromatin maintenance. Nat Rev Mol Cell Biol. 2004;5(4):296-304.

23. Chikuma S, Suita N, Okazaki IM, Shibayama S, Honjo T. TRIM28 prevents autoinflammatory T cell development in vivo. Nat Immunol. 2012;13(6):596-603.

24. Margueron R, Reinberg D. The Polycomb complex PRC2 and its mark in life. Nature. 2011;469(7330):343-349.

25. Karantanos T, Chistofides A, Barhdan K, Li L, Boussiotis VA. Regulation of T cell differentiation and function by EZH2. Front Immunol. 2016;7:172.

26. Tumes DJ, et al. The polycomb protein Ezh2 regulates differentiation and plasticity of CD4(+) T helper type 1 and type 2 cells. Immunity. 2013;39(5):819-832.

27. Zhang Y, et al. The polycomb repressive complex 2 governs life and death of peripheral T cells. Blood. 2014;124(5):737-749.

28. Yang XP, et al. EZH2 is crucial for both differentiation of regulatory T cells and T effector cell expansion. Sci Rep. 2015;5:10643

29. He S, et al. The histone methyltransferase Ezh2 is a crucial epigenetic regulator of allogeneic T-cell responses mediating graftversus-host disease. Blood. 2013;122(25):4119-4128.

30. Kelly TK, De Carvalho DD, Jones PA. Epigenetic modifications as therapeutic targets. Nat Biotechnol. 2010;28(10):1069-1078.

31. Tough DF, Tak PP, Tarakhovsky A, Prinjha RK. Epigenetic drug discovery: breaking through the immune barrier. Nat Rev Drug Discov. 2016;15(12):835-853.

32. Gulati N, Béguelin W, Giulino-Roth L. Enhancer of zeste homolog 2 (EZH2) inhibitors. Leuk Lymphoma. 2018;59(7):15741585 .

33. Johanson TM, Chan WF, Keenan CR, Allan RS. Genome organization in immune cells: unique challenges. Nat Rev Immunol. 2019;null:null.

34. Aloia L, Di Stefano B, Di Croce L. Polycomb complexes in stem cells and embryonic development. Development. 2013;140(12):2525-2534.

35. Gil J, Peters G. Regulation of the INK4b-ARF-INK4a tumour suppressor locus: all for one or one for all. Nat Rev Mol Cell Biol. 2006;7(9):667-677.

36. Jacobsen JA, et al. EZH2 regulates the developmental timing of effectors of the pre-antigen receptor checkpoints. J Immunol. 2017;198(12):4682-4691.

37. Walker C, Virchow JC, Bruijnzeel PL, Blaser K. T cell subsets and their soluble products regulate eosinophilia in allergic and nonallergic asthma. J Immunol. 1991;146(6):1829-1835.

38. Walker C, Kaegi MK, Braun P, Blaser K. Activated T cells and eosinophilia in bronchoalveolar lavages from subjects with asthma correlated with disease severity. J Allergy Clin Immunol. 1991;88(6):935-942.

39. Cammas F, Mark M, Dollé P, Dierich A, Chambon P, Losson R. Mice lacking the transcriptional corepressor TIF1beta are 
defective in early postimplantation development. Development. 2000;127(13):2955-2963.

40. Moon JJ, et al. Quantitative impact of thymic selection on Foxp3 ${ }^{+}$and Foxp3 subsets of self-peptide/MHC class II-specific CD4 ${ }^{+}$T cells. Proc Natl Acad Sci USA. 2011;108(35):14602-14607.

41. Irizarry RA, et al. Exploration, normalization, and summaries of high density oligonucleotide array probe level data. Biostatis tics. 2003;4(2):249-264.

42. Ritchie ME, et al. limma powers differential expression analyses for RNA-sequencing and microarray studies. Nucleic Acids Res. 2015;43(7):e47.

43. McCarthy DJ, Smyth GK. Testing significance relative to a fold-change threshold is a TREAT. Bioinformatics. 2009;25(6):765-771. 